\title{
RISK ARBITRAGE AND HEDGING TO ACCEPTABILITY
}

\author{
EMMANUEL LEPINETTE AND ILYA MOLCHANOV
}

\begin{abstract}
The classical discrete time model of transaction costs relies on the assumption that the increments of the feasible portfolio process belong to the solvency set at each step. We extend this setting by assuming that any such increment belongs to the sum of an element of the solvency set and the family of acceptable positions, e.g. with respect to a dynamic risk measure.

We describe the sets of superhedging prices, formulate several no risk arbitrage conditions and explore connections between them. If the acceptance sets consist of non-negative random vectors, that is the underlying dynamic risk measure is the conditional essential infimum, we extend many classical no arbitrage conditions in markets with transaction costs and provide their natural geometric interpretations. The mathematical technique relies on results for unbounded and possibly non-closed random sets in the Euclidean space.
\end{abstract}

\section{INTRODUCTION}

Transaction costs in financial markets are often described using solvency sets, which are random convex sets consisting of all financial positions (in physical quantities) regarded better than the zero position or at least equivalent to it. In the dynamic discrete time setting, the solvency sets form a set-valued random process $\left(K_{t}\right)_{t=0, \ldots, T}$ adapted to the underlying filtration $\left(\mathcal{F}_{t}\right)_{t=0, \ldots, T}$. In many cases, solvency sets are cones, and the corresponding model is known as Kabanov's model with proportional transaction costs, see $[17,25]$.

If $\xi$ is a claim that matures at time $T$, then the set of initial positions suitable as a starting value for a self-financing portfolio process $\left(V_{t}\right)_{t=0, \ldots, T}$ paying $\xi$ at maturity forms the family of superhedging prices

Date: June 16, 2016.

2010 Mathematics Subject Classification. 91G20,60D05, 60G42.

Key words and phrases. acceptance set, risk arbitrage, risk measure, superhedging, solvency set, random set, transaction costs.

IM supported by the Swiss National Science Foundation Grant 200021-153597. 
for $\xi$. The self-financing requirement amounts to the fact that the increment $V_{t-1}-V_{t}$ of the portfolio process is solvent at all times, that is a.s. belongs to $K_{t}$ for all $t$ (in this case, the increment is said to be a selection of $K_{t}$ ). This may lead to rather high superhedging prices.

The key idea of this work is to extend the family of self-financing portfolio processes by requiring that $V_{t-1}-V_{t}$ equals the sum of a selection of $K_{t}$ and another random vector that is not necessarily solvent, but is acceptable with respect to a certain dynamic risk measure. This can be heuristically viewed as "softening" of the boundary of $K_{t}$. Then $\left(V_{t}\right)_{t=0, \ldots, T}$ is called an acceptable portfolio process. In particular, the classical superhedging setting arises if the conditional essential infimum is chosen as the risk measure, so that acceptable random vectors necessarily have all a.s. non-negative components. This hedging to acceptability substantially increases the choice of possible hedging strategies, but in some cases may lead to arbitrage.

Example 1.1. Let $r$ be any coherent risk measure. Consider the one period zero interest model with two currencies as the assets. Assume that the exchange rate $\pi$ (so that $\pi$ units of the second currency buy one unit of the first one) at time one is log-normally distributed (in the real world) and the exchanges are free from transaction cost. Then the positions $\gamma^{\prime}=(-a, \pi a)$ and $\gamma^{\prime \prime}=(a,-\pi a)$ for $a>0$ are reachable from $(0,0)$ at zero costs. Their risks are $(a, \operatorname{ar}(\pi))$ and $(-a, \operatorname{ar}(-\pi))$. In order to secure the capital reserves for $\gamma^{\prime}$, the agent has to reserve $a$ of the first currency and $\operatorname{ar}(\pi)$ of the second one (note that $r(\pi)<0$ ). If the exchange rate at time zero is $\pi_{0}$, the initial cost expressed in the second currency is

$$
\pi_{0} a+\operatorname{ar}(\pi)=a\left(\pi_{0}+r(\pi)\right) .
$$

In order to secure $\gamma^{\prime \prime}$, the initial cost is $a\left(-\pi_{0}+r(-\pi)\right)$. If $\pi_{0}$ does not belong to the interval $[-r(\pi), r(-\pi)]$, then either $\pi_{0}+r(\pi)<0$ or $-\pi_{0}+r(-\pi)<0$, and we let $a$ grow to release infinite capital at time zero. Note that this model does not admit financial arbitrage, since there exists a martingale measure. This example can be modified by accounting for transaction costs.

It is accepted by now that risks of multivariate positions involving possible exchanges of assets and transaction costs are described as sets, see $[2,12]$. Indeed, the multiasset setting naturally gives rise to a possibility to offset a risky position using various combinations of assets. In this framework it is also natural to consider the family of all attainable positions as a set-valued portfolio and regard it acceptable if it contains a selection with all individually acceptable components, see [22]. 
Treating both arguments and values of a risk measure as random sets leads to law invariant risk measures and makes it possible to iterate the construction, which is essential to handle dynamic risk measures.

This paper aims to introduce geometric characterisation of superhedging prices and the corresponding no-arbitrage conditions with acceptable portfolio processes. On this way, we introduce a constructive definition of dynamic multivariate risks based on families of acceptable positions and so extend the existing works on dynamic risk measures $[6,7]$ by letting the arguments of risks and their values be subfamilies of the whole set of random vectors in $\mathbb{R}^{d}$. In many instances, these sets satisfy the decomposability property and may be interpreted as random (possibly non-closed) sets.

Section 2 introduces general random sets, their selections and treats various measurability issues, in particular, it is shown that the Minkowski (elementwise) sum of two random closed sets is measurable, no matter if the sum is closed or not. A special attention is devoted to the decomposability and infinite decomposability properties, which are the key concepts suitable to relate families of random vectors and selections of random sets. While we work with random sets in the Euclidean space, many results hold for random sets in general Banach spaces.

Each $\mathcal{F}$-measurable random closed set $X$ can be viewed as the family of its measurable selections. It is possible to restrict the latter family by considering selections measurable with respect to a sub- $\sigma$-algebra $\mathcal{H} \subset \mathcal{F}$. They define the conditional core of $X$ introduced in Section 3 . If $\mathcal{H}$ is trivial, then the conditional core becomes the set of deterministic points that belong to $X$ a.s. and is also related to the essential intersection considered in [15]. If $X$ is a.s. convex, its conditional core can be obtained by taking the conditional essential infimum of its support function. The conditional essential maximum leads to the dual concept of the conditional convex hull. While the conditional core of the sum of sets is a superset of the sum of their conditional cores, the opposite inclusion holds for the conditional convex hull.

The conditional expectation of $X$ is a random closed set generated by the conditional expectations of integrable selections of $X$, see $[16$, 21]. We extend this concept by working with generalised conditional expectations and show that it is sandwiched between the conditional core and the conditional convex hull of $X$.

We refer to [5] and [10] for the basics of static risk measures and to [1] for a survey of the dynamic $\mathcal{L}^{\infty}$-setting. The module approach worked out in $[8,9]$ provides a versatile way to handle dynamic risk measures well beyond the $\mathcal{L}^{\infty}$-setting. 
Static risk measures are usually defined on $\mathcal{L}^{p}(\mathbb{R}, \mathcal{F})$ with $p \in[1, \infty]$. However, in many cases, they are well defined also on larger sets. For example, $r(\xi)=-\operatorname{ess} \inf \xi$ makes sense for all random variables essentially bounded from below by a constant. The boundedness or integrability of their absolute values is irrelevant. Quite similarly, if $r(\xi)=-\mathrm{E} \xi$, then the acceptance set is defined as the family of $\xi$ such that their positive and negative parts satisfy $\mathrm{E} \xi^{+} \geqslant \mathrm{E} \xi^{-}>\infty$. The boundedness of $\mathrm{E} \xi^{+}$is not required.

To account for similar effects in relation to multivariate dynamic risk measures, we work with acceptance sets instead of risk measures. The acceptance sets $\mathcal{C}_{t, s}$ with $t \leqslant s$ are subsets of the sum of the family of $\mathcal{F}_{s}$-measurable random vectors in $\mathbb{R}^{d}$ that admit generalised conditional $p$ th moment with respect to $\mathcal{F}_{t}$ and the family of all $\mathcal{F}_{s}$-measurable random vectors in $\mathbb{R}_{+}^{d}$. Section 4 introduces basic conditions on the acceptance sets and several optional ones. The acceptance sets can be generated by any conventional univariate dynamic convex risk measure as the family of random vectors with individually acceptable components.

The dynamic selection risk measure $\mathrm{R}_{t, s}(\Xi)$ for a family $\Xi \subset$ $\mathcal{L}^{0}\left(\mathbb{R}^{d}, \mathcal{F}_{T}\right)$ is introduced as the $\mathcal{L}^{0}$-closure of $\left(\Xi+\mathcal{C}_{t, s}\right) \cap \mathcal{L}^{0}\left(\mathbb{R}^{d}, \mathcal{F}_{t}\right)$. If $\Xi=\mathcal{L}^{0}\left(X, \mathcal{F}_{T}\right)$ is the family of selections for a random closed set $X$, then $\mathrm{R}_{t, s}(X)=\mathrm{R}_{t, s}(\Xi)$ itself is an $\mathcal{F}_{t}$-measurable random closed set. In comparison with [7], this approach explicitly defines a set-valued risk measure instead of imposing on it some axiomatic properties. This yields a set-valued risk measure with a set-valued argument that can be naturally iterated in the dynamic framework. The conditional convexity of the acceptance sets yields that

$$
\mathrm{R}_{t, s}(\lambda X+(1-\lambda) Y) \supset \lambda \mathrm{R}_{t, s}(X)+(1-\lambda) \mathrm{R}_{t, s}(Y) \quad \text { a.s. }
$$

for any $\lambda \in \mathcal{L}^{0}\left([0,1], \mathcal{F}_{t}\right)$ and random closed sets $X$ and $Y$, that is the risk measure is also conditionally convex. The static case of this construction was considered in [22], where properties of selection risk measures in the coherent case are obtained, some of them easily extendable for a dynamic convex case. Comparing to [22], we work with solvency sets instead of portfolios available at price zero and also allow the argument of the risk measure to be a rather general family of random vectors.

The hedging to acceptability relies on a sequence $\left(K_{t}\right)_{t=0, \ldots, T}$ of solvency sets and the acceptance sets $\mathcal{C}_{t, s}$. Note that the solvency sets are not assumed to be conical, since non-conical models naturally appear, e.g. in the order book setting. An acceptable portfolio process 
$\left(V_{t}\right)_{t=0, \ldots, T}$ considered in Section 5 satisfies $V_{t-1}-V_{t}=k_{t}+\eta_{t}$ for $k_{t} \in \mathcal{L}^{0}\left(K_{t}, \mathcal{F}_{t}\right), \eta_{t} \in \mathcal{C}_{t-1, t}$, and all $t$.

If $\xi$ is a terminal claim on $d$ assets, then $\Xi_{t}^{\xi}$ denotes the set of all initial endowments at time $t$ that ensure the existence of an acceptable portfolio process paying $\xi$ at maturity, that is $V_{T} \in \xi+K_{T}$ a.s. Equivalently, $\Xi_{t}^{\xi}$ is the family of $\mathcal{F}_{t}$-measurable elements of $\left(\xi-\mathrm{A}_{t, T}\right)$, where $A_{t, T}$ is the set of claims attainable at time $T$ starting from zero investment. The set $\Xi_{t}^{\xi}$ may be used to assess the risk associated with $\xi$ at time $t$.

The classical no arbitrage conditions are imposed on the set of attainable claims, and so they may be formulated in our setting in terms of the sets $\Xi_{t}^{0}$ (that arises if $\xi=0$ ), its asymptotic variant, or using the attainable sets. These no risk arbitrage conditions are introduced and analysed in Section 6. It is shown that in some cases it is possible to represent the families of capital requirements as a set-valued process, and the no risk arbitrage conditions can be characterised in terms of weakly consistent price systems. It should be noted that the risk arbitrage only makes sense in the multiasset setting; if $K_{t}=\mathbb{R}_{+}^{d}$ (which is always the case on the line), then all no risk arbitrage conditions automatically hold. This is also the case for the classical financial arbitrage that needs at least one risky asset apart from the cash.

The sets $\mathcal{C}_{t, s}$ of acceptable positions always contain the family $\mathcal{L}^{0}\left(\mathbb{R}_{+}^{d}, \mathcal{F}_{s}\right)$ of random vectors with a.s. non-negative components and, in many cases, $\mathcal{C}_{t, s}$ is a subset of the family of random vectors with non-negative generalised conditional expectation given $\mathcal{F}_{t}$. Thus, the no risk arbitrage conditions are sandwiched between those for the risk measure based on the conditional essential infimum and on the conditional expectation. The first choice corresponds to the classical financial arbitrage with transaction costs, where our no risk arbitrage conditions become the classical ones. Section 7 recovers and extends several results from [17]. Our approach also in this case yields new geometric interpretation of the sets of superhedging prices with possibly non-conical solvency sets, where the classical consistent price systems characterisation fails. Section 8 adapts the conditional expectation as the acceptability criterion and characterises the corresponding no arbitrage conditions. These cases are illustrated on a two-asset example in Section 9. 


\section{Measurable SELECTIONS OF RANDOM SETS}

2.1. Decomposability. Let $\mathbb{R}^{d}$ be the Euclidean space with norm $\|\cdot\|$ and the Borel $\sigma$-algebra $\mathcal{B}\left(\mathbb{R}^{d}\right)$. The closure of a set $A \subset \mathbb{R}^{d}$ is denoted by $\mathrm{cl} A$ and the interior by $\operatorname{int} A$. Furthermore, let $B$ be the unit Euclidean ball centred at the origin, so that $r B$ is the ball of radius $r$.

A set-valued function $\omega \mapsto X(\omega) \subset \mathbb{R}^{d}$ from a complete probability space $(\Omega, \mathcal{F}, \mathrm{P})$ to the family of all subsets of $\mathbb{R}^{d}$ is called $\mathcal{F}$-measurable if its graph

$$
\operatorname{Gr} X=\left\{(\omega, x) \in \Omega \times \mathbb{R}^{d}: x \in X(\omega)\right\} \subset \Omega \times \mathbb{R}^{d}
$$

belongs to the product $\sigma$-algebra $\mathcal{F} \otimes \mathcal{B}\left(\mathbb{R}^{d}\right)$. In this case, $X$ is said to be a random set. In the same way the $\mathcal{H}$-measurability of $X$ with respect to a sub- $\sigma$-algebra $\mathcal{H}$ of $\mathcal{F}$ is defined. Unless otherwise stated, by the measurability we always understand the measurability with respect to $\mathcal{F}$. The random set $X$ is said to be closed if $X(\omega)$ is a closed set for almost all $\omega$.

Definition 2.1. An $\mathcal{F}$-measurable random element $\xi$ in $\mathbb{R}^{d}$ such that $\xi(\omega) \in X(\omega)$ for almost all $\omega \in \Omega$ is said to be an $\mathcal{F}$-measurable selection (selection in short) of $X, \mathcal{L}^{0}(X, \mathcal{F})$ denotes the family of all $\mathcal{F}$ measurable selections of $X$, and $\mathcal{L}^{p}(X, \mathcal{F})$ is the family of $p$-integrable ones.

It is known that an a.s. non-empty random set has at least one selection, see [14, Th. 4.4]. Note that $\mathcal{L}^{0}\left(\mathbb{R}^{d}, \mathcal{F}\right)$ is equipped with the topology generated by the convergence in probability. The closure in the strong topology in $\mathcal{L}^{p}$ for $p \in[1, \infty)$ is denoted by $\mathrm{cl}_{p}$ and $\mathrm{cl}_{0}$ is the closure in probability. If $p=\infty$, the closure is considered in the $\sigma\left(\mathcal{L}^{\infty}, \mathcal{L}^{1}\right)$-topology, equivalently with respect to a.s. convergence of bounded sequences.

Definition 2.2. A family $\Xi \subset \mathcal{L}^{0}\left(\mathbb{R}^{d}, \mathcal{F}\right)$ is said to be infinitely $\mathcal{H}$ decomposable if

$$
\sum_{n} \xi_{n} \mathbf{1}_{A_{n}} \in \Xi
$$

for all sequences $\left(\xi_{n}\right)_{n \geqslant 1}$ from $\Xi$ and all $\mathcal{H}$-measurable partitions $\left(A_{n}\right)_{n \geqslant 1}$ of $\Omega ; \Xi$ is $\mathcal{H}$-decomposable if this holds for finite partitions.

The decomposable subsets of $\mathcal{L}^{0}\left(\mathbb{R}^{d}, \mathcal{F}\right)$ are called stable and infinitely decomposable ones are called $\sigma$-stable in [3]. Observe that an infinitely decomposable set is not necessarily closed in $\mathcal{L}^{0}$, while its closure in $\mathcal{L}^{0}$ is infinitely decomposable. The following result is well known for $p=1$ [16], for $p \in[1, \infty]$ [21, Th. 2.1.6], and is mentioned in [17, Prop. 5.4.3] for $p=0$ and $\mathcal{H}=\mathcal{F}$. The proof follows the scheme of 
$\left[21\right.$, Th. 2.1.6] by replacing the $\mathcal{L}^{p}$-metric with the metric $\mathrm{E}[\|\xi-\eta\| \wedge 1]$ that corresponds to the convergence in probability and relying on the subsequent Lemma 2.4.

Theorem 2.3. Let $\Xi$ be a non-empty subset of $\mathcal{L}^{p}\left(\mathbb{R}^{d}, \mathcal{F}\right)$ for $p=0$ or $p \in[1, \infty]$. Then

$$
\Xi \cap \mathcal{L}^{p}\left(\mathbb{R}^{d}, \mathcal{H}\right)=\mathcal{L}^{p}(X, \mathcal{H})
$$

for an $\mathcal{H}$-measurable random closed set $X$ if and only if $\Xi$ is $\mathcal{H}$ decomposable and closed.

Lemma 2.4. Let $\xi_{n} \in \mathcal{L}^{0}\left(\mathbb{R}^{d}, \mathcal{F}\right), n \geqslant 1$, so that $X(\omega)=\operatorname{cl}\left\{\xi_{n}(\omega), n \geqslant\right.$ $1\}$ is a random closed set. Let $\xi \in \mathcal{L}^{0}(X, \mathcal{F})$. Then, for each $\varepsilon>0$, there exists a measurable partition $A_{1}, \ldots, A_{n}$ of $\Omega$ such that

$$
\mathrm{E}\left[\left\|\xi-\sum_{i=1}^{n} \mathbf{1}_{A_{i}} \xi_{i}\right\| \wedge 1\right] \leqslant \varepsilon .
$$

Proposition 2.5. Let $\Xi$ be infinitely $\mathcal{F}$-decomposable, and let $X$ be an $\mathcal{F}$-measurable a.s. nonempty random open set. Then

$$
\mathcal{L}^{0}(X, \mathcal{F})+\Xi=\mathcal{L}^{0}(X, \mathcal{F})+\operatorname{cl}_{0} \Xi .
$$

Proof. Consider $\gamma \in \mathcal{L}^{0}(X, \mathcal{F})$ and $\xi \in \mathrm{cl}_{0} \Xi$, so that $\xi_{n} \rightarrow \xi$ a.s. for $\xi_{n} \in \Xi, n \geqslant 1$. By a measurable selection argument, there exists $\alpha \in \mathcal{L}^{0}((0, \infty), \mathcal{F})$ such that the ball of radius $\alpha$ centred at $\gamma$ is a subset of $X$ a.s. Let us define, up to a null set, the $\mathcal{F}$-measurable mapping

$$
k(\omega)=\inf \left\{n:\left\|\xi(\omega)-\xi_{n}(\omega)\right\| \leqslant \alpha(\omega)\right\}, \quad \omega \in \Omega .
$$

Then

$$
\hat{\xi}(\omega)=\xi_{k(\omega)}(\omega)=\sum_{j=1}^{\infty} \xi_{j} 1_{k(\omega)=j},
$$

is also $\mathcal{F}$-measurable and belongs to $\Xi$ by assumption. Since $\|\hat{\xi}-\xi\| \leqslant \alpha$ a.s., $\xi+\gamma=(\xi+\gamma-\hat{\xi})+\hat{\xi} \in \mathcal{L}^{0}(X, \mathcal{F})+\Xi$.

Proposition 2.6. If $X$ is a random set, then its pointwise closure cl $X(\omega), \omega \in \Omega$, is a random closed set, and $\mathcal{L}^{0}(\operatorname{cl} X, \mathcal{F})=\operatorname{cl}_{0} \mathcal{L}^{0}(X, \mathcal{F})$. Furthermore, there exists a countable family $\left(\xi_{i}\right)_{i \geqslant 1}$ of measurable selections of $X$ such that $\mathrm{cl} X=\operatorname{cl}\left\{\xi_{i}, i \geqslant 1\right\}$ a.s.

Proof. Since the probability space is complete and the graph of $X$ is measurable in the product space, the projection theorem yields that $\{X \cap G \neq \varnothing\} \in \mathcal{F}$ for any open set $G$. Finally, note that $X$ hits any open set $G$ if and only if $\operatorname{cl} X$ hits $G$. Thus, cl $X$ is Effros measurable and so is a random closed set, see [21, Sec. 1.2.1]. 
The inclusion $\mathrm{cl}_{0} \mathcal{L}^{0}(X, \mathcal{F}) \subset \mathcal{L}^{0}(\mathrm{cl} X, \mathcal{F})$ obviously holds. Since $\mathrm{cl}_{0} \mathcal{L}^{0}(X, \mathcal{F})$ is decomposable, there exists a random closed set $Y$ such that $\mathrm{cl}_{0} \mathcal{L}^{0}(X, \mathcal{F})=\mathcal{L}^{0}(Y, \mathcal{F})$. Since $\mathcal{L}^{0}(X, \mathcal{F}) \subset \mathcal{L}^{0}(Y, \mathcal{F})$, we have $X \subset Y$ a.s. Therefore, cl $X \subset Y$ a.s. and the conclusion follows.

The existence of a countable respresentation of $\mathrm{cl} X$ is well known, see e.g. [21, Th. 2.2.3]. It is called the Castaing representation.

\subsection{Measurable versions and Minkowski sums.}

Proposition 2.7. For any closed set-valued mapping $X(\omega), \omega \in \Omega$, there exists a random closed set $Y$ (called the measurable version of $X)$ such that $\mathcal{L}^{0}(X, \mathcal{F})=\mathcal{L}^{0}(Y, \mathcal{F})$.

Proof. Assume that $\mathcal{L}^{0}(X, \mathcal{F})$ is non-empty, otherwise $Y=\varnothing$ a.s. Since $\mathcal{L}^{0}(X, \mathcal{F})$ is closed and decomposable, Theorem 2.3 ensures the existence of $Y$ that satisfies the required conditions.

For $A_{1}, A_{2} \subset \mathbb{R}^{d}$, define their pointwise (Minkowski) sum as

$$
A_{1}+A_{2}=\left\{x_{1}+x_{2}: x_{1} \in A_{1}, x_{2} \in A_{2}\right\} .
$$

The same definition applies to the sum of subsets of $\mathcal{L}^{0}\left(\mathbb{R}^{d}, \mathcal{F}\right)$. The set of pairwise differences of points from $A_{1}$ and $A_{2}$ is obtained as $A_{1}+\left(-A_{2}\right)$, or shortly $A_{1}-A_{2}$, where $-A_{2}=\left\{-x: x \in A_{2}\right\}$ is the centrally symmetric variant of $A_{2}$. For the sum $A+\{x\}$ of a set and a singleton we write shortly $A+x$. Note that the sum of two closed sets is not necessarily closed, while it becomes closed if at least one of the closed summands is compact. The following result differs from [21, Th. 2.2.25] in considering the possibly non-closed sum of two random closed sets.

Lemma 2.8. Let $X_{1}$ and $X_{2}$ be two random sets. Then $\mathcal{L}^{0}\left(X_{1}, \mathcal{F}\right)+$ $\mathcal{L}^{0}\left(X_{2}, \mathcal{F}\right)=\mathcal{L}^{0}\left(X_{1}+X_{2}, \mathcal{F}\right)$. If both $X_{1}$ and $X_{2}$ are random closed sets, then $X_{1}+X_{2}$ is measurable.

Proof. It is trivial that $\mathcal{L}^{0}\left(X_{1}, \mathcal{F}\right)+\mathcal{L}^{0}\left(X_{2}, \mathcal{F}\right) \subset \mathcal{L}^{0}\left(X_{1}+X_{2}, \mathcal{F}\right)$. To prove the reverse inclusion, consider $\gamma \in \mathcal{L}^{0}\left(X_{1}+X_{2}, \mathcal{F}\right)$. Since $X_{1}$ and $X_{2}$ are $\mathcal{F}$-measurable, the measurable selection theorem [17, Th. 5.4.1] yields that there exist $\mathcal{F}$-measurable selections $\xi^{\prime} \in \mathcal{L}^{0}\left(X_{1}, \mathcal{F}\right)$ and $\xi^{\prime \prime} \in \mathcal{L}^{0}\left(X_{2}, \mathcal{F}\right)$ such that $\xi=\xi^{\prime}+\xi^{\prime \prime}$.

Now assume that $X_{1}$ and $X_{2}$ are closed and consider their Castaing representations $X_{1}(\omega)=\operatorname{cl}\left\{\xi_{i}^{\prime}(\omega), i \geqslant 1\right\}$ and $X_{2}(\omega)=\operatorname{cl}\left\{\xi_{i}^{\prime \prime}(\omega), i \geqslant 1\right\}$. The measurability of $X_{1}+X_{2}$ follows from the representation

$$
\operatorname{Gr}\left(X_{1}+X_{2}\right)
$$




$$
=\bigcup_{k \geqslant 1} \bigcap_{m \geqslant 1} \bigcup_{i, j \geqslant 1}\left\{(\omega, x):\left\|x-\xi_{i}^{\prime}(\omega)-\xi_{j}^{\prime \prime}(\omega)\right\| \leqslant \frac{1}{m},\left\|\xi_{i}^{\prime}(\omega)\right\| \leqslant k\right\} .
$$

Indeed, $\operatorname{Gr}\left(X_{1}+X_{2}\right)$ is clearly contained in the right-hand side of (2.1), while the reverse inclusion is shown by a compactness argument.

Proposition 2.9. If $X$ is a random set, then the pointwise closure of its convex hull co $X$ admits a measurable version.

Proof. By Proposition 2.7, the closure of co $X$ admits a measurable version $Y$. Then $\mathcal{L}^{0}(Y, \mathcal{F})$ is the set of all random variables taking values a.s. in the pointwise closure of co $X$. In particular, $\mathcal{L}^{0}(Y, \mathcal{F})$ is closed and convex. It suffices to show that $Y$ is a.s. convex. Assume that this is not the case. Since the set

$$
\left\{(w, \alpha, x, y) \in \Omega \times[0,1] \times\left(\mathbb{R}^{d}\right)^{2}: x, y \in Y, \alpha x+(1-\alpha) y \notin Y\right\}
$$

is $\mathcal{F} \otimes \mathcal{B}([0,1]) \otimes \mathcal{B}\left(\left(\mathbb{R}^{d}\right)^{2}\right)$-measurable, we may construct a measurable version of this set at least on a non-null set. We obtain a contradiction, since $\mathcal{L}^{0}(Y, \mathcal{F})$ is convex.

2.3. Support function. If $X$ is a random set in $\mathbb{R}^{d}$, then its support function is defined by

$$
h_{X}(u)=\sup \{\langle u, x\rangle: x \in X\}, \quad u \in \mathbb{R}^{d},
$$

where $\langle u, x\rangle$ denotes the scalar product. If $X(\omega)$ is empty, then the support function is set to be $-\infty$. It is easy to see that the support function does not discern between the random set and a measurable version of its closed convex hull.

Let $X$ be a random closed convex set, that is $X(\omega)$ is convex for almost all $\omega$. It is well known that its support function $h_{X}(u)$ is a random variable in $\overline{\mathbb{R}}=[-\infty, \infty]$ for each $u \in \mathbb{R}^{d}$. The following result extends this and (2.2) for random $u$. It refers to the definition of the essential supremum from Appendix A.

Lemma 2.10. For every $\zeta \in \mathcal{L}^{0}\left(\mathbb{R}^{d}, \mathcal{F}\right)$ and random closed convex set $X, h_{X}(\zeta)$ is a random variable in $[-\infty, \infty]$, and

$$
h_{X}(\zeta)=\operatorname{ess}_{\sup _{\mathcal{F}}}\left\{\langle\zeta, \xi\rangle: \xi \in \mathcal{L}^{0}(X, \mathcal{F})\right\} \quad \text { a.s. }
$$

if $X$ is a.s. non-empty.

Proof. Since $\{X=\varnothing\} \in \mathcal{F}$, it is possible to assume that $X$ is a.s. non-empty. By Proposition 2.6, $X=\operatorname{cl}\left\{\xi_{i}, i \geqslant 1\right\}$, so that $h_{X}(\zeta)=$ $\sup _{i}\left\langle\zeta, \xi_{i}\right\rangle$ is $\mathcal{F}$-measurable. It is immediate that $h_{X}(\zeta) \geqslant\langle\zeta, \xi\rangle$ for all $\xi \in \mathcal{L}^{0}(X, \mathcal{F})$, so that

$$
h_{X}(\zeta) \geqslant \operatorname{ess}_{\sup }\left\{\langle\zeta, \xi\rangle: \xi \in \mathcal{L}^{0}(X, \mathcal{F})\right\} .
$$


Assume that $X$ is a.s. compact, that is $\left|h_{X}(\zeta)\right|<\infty$ a.s. For any $\varepsilon>0$, the random closed set $X \cap\left\{x:\langle x, \zeta\rangle \geqslant h_{X}(\zeta)-\varepsilon\right\}$ is a.s. non-empty and so possesses a selection $\eta$. Then

$$
\operatorname{ess}_{\sup }\left\{\langle\zeta, \xi\rangle: \xi \in \mathcal{L}^{0}(X, \mathcal{F})\right\} \geqslant\langle\zeta, \eta\rangle \geqslant h_{X}(\zeta)-\varepsilon .
$$

Letting $\varepsilon \downarrow 0$ yields (2.3). For a general closed set $X, h_{X}(\zeta)$ is the limit of $h_{X^{n}}(\zeta)$ as $n \rightarrow \infty$, where $X^{n}=X \cap n B$. Since (2.3) holds for $X^{n}$ and $X^{n} \subset X$ a.s.,

$$
h_{X}(\zeta)=\lim _{n} h_{X^{n}}(\zeta) \leqslant \operatorname{ess} \sup _{\mathcal{F}}\left\{\langle\zeta, \xi\rangle: \xi \in \mathcal{L}^{0}(X, \mathcal{F})\right\} .
$$

\section{Conditional CORE And COnditional CONVEX hull}

3.1. Conditional core. Let $\mathcal{H}$ be a sub- $\sigma$-algebra of $\mathcal{F}$.

Definition 3.1. The $\mathcal{H}$-core (also called conditional core), $\mathbf{m}(X \mid \mathcal{H})$, of a set-valued mapping $X$ is the largest $\mathcal{H}$-measurable random set $X^{\prime}$ such that $X^{\prime} \subset X$ a.s.

The existence of the $\mathcal{H}$-core is the issue of the existence of the largest $\mathcal{H}$-measurable subset of $X$. It does not prevent $\mathbf{m}(X \mid \mathcal{H})$ from being empty. For example, if $X=\{\xi\}$ is a singleton, then $\mathbf{m}(X \mid \mathcal{H})$ exists and is empty on a non null set unless $\xi$ is $\mathcal{H}$-measurable. If $\mathcal{H}$ is the trivial $\sigma$-algebra, then $\mathbf{m}(X \mid \mathcal{H})$ is the set of all points $x \in \mathbb{R}^{d}$ such that $x \in X(\omega)$ for almost all $\omega$. Such points are called fixed points of a random set and it is obvious that the set of fixed points may be empty.

Lemma 3.2. If $\mathbf{m}(X \mid \mathcal{H})$ exists, then $\mathcal{L}^{0}(X, \mathcal{H})=\mathcal{L}^{0}(\mathbf{m}(X \mid \mathcal{H}), \mathcal{H})$, in particular $\mathbf{m}(X, \mathcal{H}) \neq \varnothing$ a.s. if and only if $\mathcal{L}^{0}(X, \mathcal{H}) \neq \varnothing$.

Proof. In order to show the non-trivial inclusion, consider $\gamma \in$ $\mathcal{L}^{0}(X, \mathcal{H})$. The random set $X^{\prime}=\{\gamma\}$ is $\mathcal{H}$-measurable and satisfies $X^{\prime} \subset X$ a.s., that is $\gamma \in \mathbf{m}(X \mid \mathcal{H})$ a.s.

Lemma 3.3. If $X$ is a random closed set, then $\mathbf{m}(X \mid \mathcal{H})$ exists and is a random closed set, which is a.s. convex if $X$ is a.s. convex.

Proof. By Theorem 2.3, $\mathcal{L}^{0}(X, \mathcal{H})=\mathcal{L}^{0}(Y, \mathcal{H})$ for an $\mathcal{H}$-measurable random closed set $Y$. Moreover, $Y=\operatorname{cl}\left\{\xi_{n}, n \geqslant 1\right\}$ a.s. for $\xi_{n} \in$ $\mathcal{L}^{0}(X, \mathcal{H}), n \geqslant 1$. Since $X$ is closed, $Y \subset X$ a.s. Since any $\mathcal{H}$ measurable random set $Z \subset X$ satisfies $\mathcal{L}^{0}(Z, \mathcal{H}) \subset \mathcal{L}^{0}(Y, \mathcal{H})$, we have $Z \subset X$ a.s., so that $Y=\mathbf{m}(X \mid \mathcal{H})$.

If $X$ is convex, then the closed convex hull of $\mathbf{m}(X \mid \mathcal{H})$ is a random closed set (see Proposition 2.9) that is contained in $X$. Thus, $\mathbf{m}(X \mid \mathcal{H})$ is a.s. convex since, being the largest $\mathcal{H}$-measurable closed subset of $X$, it coincides with the closure of its convex hull. 
Example 3.4. If $X$ is defined on a finite binomial tree, then the conditional core of $X$ is given by the intersection of the sets $X(\omega)$ for $\omega$ corresponding to the end-points of all branches that originate from a particular node of the tree.

It is easy to see that if $\mathbf{m}(X \mid \mathcal{H}), \mathbf{m}(Y \mid \mathcal{H})$, and $\mathbf{m}(X+Y \mid \mathcal{H})$ exist for set-valued mappings $X$ and $Y$, then

$$
\mathbf{m}(X \mid \mathcal{H})+\mathbf{m}(Y \mid \mathcal{H}) \subset \mathbf{m}(X+Y \mid \mathcal{H}) .
$$

Lemma 3.5. Let $X$ be an $\mathcal{F}$-measurable random set.

(i) $\lambda X$ is an $\mathcal{F}$-measurable random set for any $\lambda \in \mathcal{L}^{0}(\mathbb{R}, \mathcal{F})$.

(ii) If $\mathbf{m}(X \mid \mathcal{H})$ exists and $\lambda \in \mathcal{L}^{0}(\mathbb{R}, \mathcal{H})$, then $\mathbf{m}(\lambda X \mid \mathcal{H})$ exists and

$$
\mathbf{m}(\lambda X \mid \mathcal{H})=\lambda \mathbf{m}(X \mid \mathcal{H}) \text {. }
$$

Proof. (i) Since

$$
\operatorname{Gr}(\lambda X)=(\{\lambda=0\} \times\{0\}) \cup\left(\operatorname{Gr}(\lambda X) \cap\left(\{\lambda \neq 0\} \times \mathbb{R}^{d}\right)\right),
$$

it suffices to assume that $\lambda \neq 0$ a.s. The measurability of $\lambda X$ is immediate, since the map $\phi:(\omega, x) \mapsto\left(\omega, \lambda^{-1} x\right)$ is measurable and $\operatorname{Gr}(\lambda X)=\phi^{-1}(\operatorname{Gr} X)$.

(ii) Observe that $\lambda \mathbf{m}(X \mid \mathcal{H})$ is $\mathcal{H}$-measurable if $\lambda \in \mathcal{L}^{0}(\mathbb{R}, \mathcal{H})$ and $\lambda \mathbf{m}(X \mid \mathcal{H}) \subset \lambda X$. Suppose that $X^{\prime} \subset \lambda X$ is $\mathcal{H}$-measurable. Then

$$
X^{\prime \prime}=\lambda^{-1} X^{\prime} \mathbf{1}_{\lambda \neq 0}+X \mathbf{1}_{\lambda=0} \subset X,
$$

and $X^{\prime \prime}$ is $\mathcal{H}$-measurable. Therefore, $X^{\prime \prime} \subset \mathbf{m}(X \mid \mathcal{H})$, so that $X^{\prime} \subset$ $\lambda \mathbf{m}(X \mid \mathcal{H})$. Thus, $\mathbf{m}(\lambda X \mid \mathcal{H})$ exits and (3.2) holds.

Lemma 3.6. If $\mathcal{L}^{p}(X, \mathcal{H}) \neq \varnothing$ for $p \in[1, \infty]$ and a random closed set $X$, then $\mathcal{L}^{p}(\mathbf{m}(X \mid \mathcal{H}), \mathcal{H})=\mathcal{L}^{p}(X, \mathcal{H})$.

Proof. By the condition, $\mathbf{m}(X \mid \mathcal{H})$ admits a $p$-integrable selection, and so has the Castaing representation consisting of $p$-integrable selections, see [16] and [21].

Remark 3.7. Consider a filtration $\left(\mathcal{F}_{t}\right)_{t=0, \ldots, T}$ on $(\Omega, \mathcal{F}, \mathrm{P})$. Let $X \subset \mathbb{R}^{d}$ be a random closed set. Then the set-valued process $X_{t}=\mathbf{m}\left(X \mid \mathcal{F}_{t}\right)$, $t=0, \ldots, T$, satisfies $X_{s}=\mathbf{m}\left(X_{t} \mid \mathcal{F}_{s}\right)$ for all $s \leqslant t$.

3.2. Random convex sets and conditional convex hulls. It is possible to relate $\mathcal{H}$-cores of random closed convex sets to the conditional essential infimum of their support functions. We denote by $\mathbb{Q}$ the set of all rational numbers.

Proposition 3.8. Let $X$ be a random closed convex set such that $\mathbf{m}(X \mid \mathcal{H}) \neq \varnothing$ a.s. Then

$$
h_{\mathbf{m}(X \mid \mathcal{H})}(\zeta) \leqslant \operatorname{essinf}_{\mathcal{H}} h_{X}(\zeta), \quad \zeta \in \mathcal{L}^{0}\left(\mathbb{R}^{d}, \mathcal{H}\right) .
$$


If $X$ is a.s. bounded, then

$$
\mathbf{m}(X \mid \mathcal{H})=\bigcap_{u \in \mathbb{Q}^{d}}\left\{x \in \mathbb{R}^{d}:\langle u, x\rangle \leqslant \operatorname{essinf}_{\mathcal{H}} h_{X}(u)\right\}
$$

Proof. Fix any $\zeta \in \mathcal{L}^{0}\left(\mathbb{R}^{d}, \mathcal{H}\right)$. By Lemma 2.10 and Lemma 3.6,

$$
h_{\mathbf{m}(X \mid \mathcal{H})}(\zeta)=\operatorname{ess} \sup _{\mathcal{H}}\left\{\langle\zeta, \xi\rangle: \xi \in \mathcal{L}^{0}(X, \mathcal{H})\right\} .
$$

Moreover, $\mathbf{m}(X \mid \mathcal{H}) \subset X \subset\left\{x:\langle x, \zeta\rangle \leqslant h_{X}(\zeta)\right\}$. Thus, $\langle\zeta, \xi\rangle \leqslant h_{X}(\zeta)$ for $\xi \in \mathcal{L}^{0}(X, \mathcal{H})$. Since $\langle\zeta, \xi\rangle$ is $\mathcal{H}$-measurable, $\langle\zeta, \xi\rangle \leqslant \operatorname{essinf}_{\mathcal{H}} h_{X}(\zeta)$, so that (3.3) holds.

Assume that $X$ is a.s. bounded. The right-hand side of (3.4) is $\mathcal{H}$ measurable random convex closed set denoted by $\tilde{X}$. Since $\mathbf{m}(X \mid \mathcal{H})$ and $X$ are closed and convex, (3.3) yields that $\mathbf{m}(X \mid \mathcal{H}) \subset \tilde{X}$. Observe that $\gamma \in \mathcal{L}^{0}(\tilde{X}, \mathcal{F})$ implies $\langle u, \gamma\rangle \leqslant h_{X}(u)$ a.s. for all $u \in \mathbb{Q}^{d}$. Since $h_{X}$ is a.s. finite and continuous, $\langle u, \gamma\rangle \leqslant h_{X}(u)$ for all $u \in \mathbb{R}^{d}$ a.s., that is $\tilde{X} \subset X$ a.s. Therefore, $\tilde{X} \subset \mathbf{m}(X \mid \mathcal{H})$.

Example 3.9. The inequality in (3.3) can be strict. Let $X$ be a line in the plane passing through the origin with the non $\mathcal{H}$-measurable normal vector having a non-atomic distribution. Assume that $\mathcal{H}$ contains all null-events from $\mathcal{F}$. Then the only $\mathcal{H}$-measurable selection of $X$ is the origin, so that the left-hand side of (3.3) vanishes. For each deterministic (and so $\mathcal{H}$-measurable) non-vanishing $\zeta=u$, we have $h_{X}(u)=\infty$ a.s., so that the the right-hand side of (3.3) is infinite.

Taking conditional essential supremum of the support function yields the dual concept of the conditional convex hull $\mathbf{M}(X \mid \mathcal{H})$ of $X$.

Proposition 3.10. Let $X$ be an a.s. non-empty random closed set. Then there exists the smallest $\mathcal{H}$-measurable random convex closed set (denoted by $\mathbf{M}(X \mid \mathcal{H})$ ) that contains $X$ a.s. If $\mathbf{M}(X \mid \mathcal{H})$ is a.s. bounded,

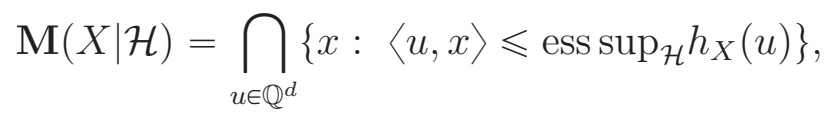

and $h_{\mathbf{M}(X \mid \mathcal{H})}(u)=\operatorname{ess} \sup _{\mathcal{H}} h_{X}(u)$ for every $u \in \mathbb{Q}^{d}$.

Proof. Assume that $X \subset c B$ a.s. for a constant $c$. Denote the righthand side of $(3.5)$ by $\tilde{X}$. Since $h_{X}(u) \leqslant \operatorname{ess} \sup _{\mathcal{H}} h_{X}(u)$ for all $u \in \mathbb{Q}^{d}$ a.s., $X \subset \tilde{X}$ a.s. Assume that $Y$ is any $\mathcal{H}$-measurable closed convex set containing $X$. We suppose without loss of generality that $X \subset Y$ on $\Omega$ and $Y \subset c B$, so that $h_{Y}$ is continuous. Since $h_{X}(u) \leqslant h_{Y}(u)$ for all $u \in \mathbb{Q}^{d}$, and $h_{Y}(u)$ is $\mathcal{H}$-measurable, $\operatorname{ess}_{\sup } h_{\mathcal{H}}(u) \leqslant h_{Y}(u)$.

If the definition of $\tilde{X}$ is modified by replacing $\operatorname{ess} \sup _{\mathcal{H}} h_{X}(u)$ for each $u \in \mathbb{Q}^{d}$ on a negligible set with $h_{Y}(u)$, then $\mathcal{L}^{0}(\tilde{X}, \mathcal{H})$ does not change. 
We then suppose without loss of generality that $\operatorname{ess}_{\sup } h_{X}(u) \leqslant h_{Y}(u)$ on $\Omega$ for every $u \in \mathbb{Q}^{d}$, so that $\tilde{X} \subset Y$ by the continuity of $h_{Y}$. Thus, $\tilde{X}$ is the smallest $\mathcal{H}$-measurable random convex closed set that contains $X$. If $X$ is not necessarily bounded, consider $X^{n}=X \cap n B$ for $n \geqslant 1$. Then

$$
\mathbf{M}(X \mid \mathcal{H})=\mathrm{cl} \bigcup_{n \geqslant 1} \mathbf{M}\left(X^{n} \mid \mathcal{H}\right)
$$

is the smallest $\mathcal{H}$-measurable random convex closed set that contains $X$ a.s. Indeed, if $X \subset Y$, then $X^{n} \subset Y$. Thus, $\mathbf{M}\left(X^{n} \mid \mathcal{H}\right) \subset Y$ for all $n$ and finally $\mathbf{M}(X \mid \mathcal{H}) \subset Y$. Therefore,

$$
\mathbf{M}(X \mid \mathcal{H}) \subset \bigcap_{u \in \mathbb{Q}^{d}}\left\{x:\langle u, x\rangle \leqslant \operatorname{ess} \sup _{\mathcal{H}} h_{X}(u)\right\} .
$$

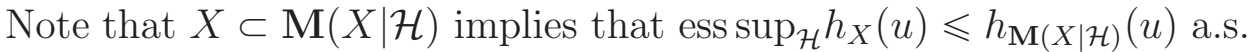
Observe that $\operatorname{ess}_{\sup } h_{X}(u)$ for each $u \in \mathbb{Q}^{d}$ can be modified on an $\mathcal{H}$ measurable negligible set, so that the latter inequality holds everywhere on $\Omega$. By construction of $\mathbf{M}(X \mid \mathcal{H})$, for $u \in \mathbb{Q}^{d}$,

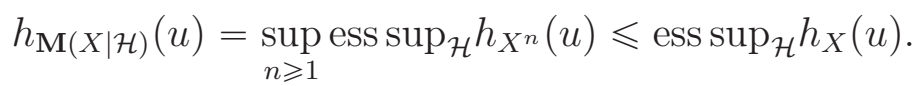

Hence $h_{\mathbf{M}(X \mid \mathcal{H})}(u)=\operatorname{ess}_{\sup _{\mathcal{H}}} h_{X}(u)$ for every $u \in \mathbb{Q}^{d}$.

If $\mathbf{M}(X \mid \mathcal{H})$ is bounded a.s., $h_{\mathbf{M}(X \mid \mathcal{H})}$ is continuous, so that $\operatorname{ess}^{\sup _{\mathcal{H}}} h_{X}(u) \leqslant h_{\mathbf{M}(X \mid \mathcal{H})}(u)$, yields that the right-hand side of (3.6) is contained in $\mathbf{M}(X \mid \mathcal{H})$, i.e. the equality holds.

3.3. Conditional expectation. The following definition relies on the concept of the generalised expectation discussed in Appendix B.

Definition 3.11. Let $X$ be a random closed set and let $\mathcal{H}$ be a sub$\sigma$-algebra of $\mathcal{F}$ such that $\mathcal{L}_{\mathcal{H}}^{1}(X, \mathcal{F}) \neq \varnothing$. The generalised conditional expectation $\mathrm{E}^{g}(X \mid \mathcal{H})$ is the $\mathcal{H}$-measurable random closed set such that

$$
\mathcal{L}^{0}\left(\mathrm{E}^{g}(X \mid \mathcal{H}), \mathcal{H}\right)=\operatorname{cl}_{0}\left\{\mathrm{E}^{g}(\xi \mid \mathcal{H}): \xi \in \mathcal{L}_{\mathcal{H}}^{1}(X, \mathcal{F})\right\} .
$$

The generalised conditional expectation $\mathrm{E}^{g}(\Xi \mid \mathcal{H})$ of a family $\Xi \subset$ $\mathcal{L}^{0}\left(\mathbb{R}^{d}, \mathcal{F}\right)$ is defined as above by taking $\xi \in \mathcal{L}_{\mathcal{H}}^{1}\left(\mathbb{R}^{d}, \mathcal{F}\right) \cap \Xi$; this applies also to the case when $\Xi$ is the family of selections of a non-closed random set.

If $\mathcal{L}^{1}(X, \mathcal{F}) \neq \varnothing$, then $X$ is said to be integrable and its conditional expectation $\mathrm{E}(X \mid \mathcal{H})$ is the random closed set such that

$$
\mathcal{L}^{1}(\mathrm{E}(X \mid \mathcal{H}), \mathcal{H})=\operatorname{cl}_{1}\left\{\mathrm{E}(\xi \mid \mathcal{H}): \xi \in \mathcal{L}^{1}(X, \mathcal{F})\right\} .
$$

It is known [16] and [21, Th. 2.1.47] that $h_{\mathrm{E}(X \mid \mathcal{H})}(u)=\mathrm{E}\left(h_{X}(u) \mid \mathcal{H}\right)$ a.s. for all $u \in \mathbb{R}^{d}$ if $X$ is a.s. convex. 
Lemma 3.12. If $X$ is integrable, $\mathcal{L}^{0}(\mathrm{E}(X \mid \mathcal{H}), \mathcal{H})$ coincides with the $\mathcal{L}^{0}$-closure of the set $\left\{\mathrm{E}(\xi \mid \mathcal{H}): \xi \in \mathcal{L}^{1}(X, \mathcal{F})\right\}$.

Proof. By definition, $\Xi=\left\{\mathrm{E}(\xi \mid \mathcal{H}): \xi \in \mathcal{L}^{1}(X, \mathcal{F})\right\}$ is included in $\mathcal{L}^{0}(\mathrm{E}(X \mid \mathcal{H}), \mathcal{H})$ which is closed in $\mathcal{L}^{0}$ since $\mathrm{E}(X \mid \mathcal{H})$ is a.s. closed. Therefore, $\mathrm{cl}_{0} \Xi \subset \mathcal{L}^{0}(\mathrm{E}(X \mid \mathcal{H}), \mathcal{H})$. By Proposition 2.6, $\mathrm{E}(X \mid \mathcal{H})=$ $\operatorname{cl}\left\{\xi_{i}, i \geqslant 1\right\}$, where $\xi_{i} \in \operatorname{cl}_{1} \Xi$ for all $i \geqslant 1$. Then $\left\{\xi_{i}, i \geqslant 1\right\} \subset \operatorname{cl}_{0} \Xi$, so that $\mathcal{L}^{0}(\mathrm{E}(X \mid \mathcal{H}), \mathcal{H}) \subset \mathrm{cl}_{0} \Xi$ by Lemma 2.4 .

Lemma 3.13. If $X$ is an integrable random closed set, then $\mathrm{E}(X \mid \mathcal{H})=$ $\mathrm{E}^{g}(X \mid \mathcal{H})$.

Proof. To show the non-trivial inclusion, consider $\xi \in \mathcal{L}_{\mathcal{H}}^{1}(X, \mathcal{F})$ and the corresponding partition $\left(B_{i}\right)_{i \geqslant 1}$. If $\gamma \in \mathcal{L}^{1}(X, \mathcal{F})$, then

$$
\mathrm{E}^{g}(\xi \mid \mathcal{H})=\lim _{n \rightarrow \infty} \sum_{i=1}^{n}\left[\mathrm{E}\left(\xi \mathbf{1}_{B_{i}} \mid \mathcal{H}\right) \mathbf{1}_{B_{i}}+\mathrm{E}(\gamma \mid \mathcal{H}) 1_{\Omega \backslash \cup_{i \leqslant n} B_{i}}\right] \quad \text { a.s. }
$$

Since $\xi \mathbf{1}_{B_{i}}$ and $\gamma$ are integrable and $\mathrm{E}(X \mid \mathcal{H})$ is $\mathcal{H}$-decomposable, the sum under the limit belongs to $\mathrm{E}(X \mid \mathcal{H})$. Therefore, $\mathrm{E}^{g}(\xi \mid \mathcal{H}) \in$ $\operatorname{cl}_{0} \mathcal{L}^{0}(\mathrm{E}(X \mid \mathcal{H}), \mathcal{H})$, where the latter set is closed in $\mathcal{L}^{0}$ by Lemma 3.12. Thus, $\mathrm{E}^{g}(X \mid \mathcal{H}) \subset \mathrm{E}(X \mid \mathcal{H})$ a.s.

Lemma 3.14. Let $X$ be a random closed set such that $\mathcal{L}_{\mathcal{H}}^{1}(X, \mathcal{F}) \neq \varnothing$. Then, for every $\xi \in \mathcal{L}_{\mathcal{H}}^{1}(X, \mathcal{F}), X-\xi$ is integrable and

$$
\mathrm{E}^{g}(X \mid \mathcal{H})=\mathrm{E}(X-\xi \mid \mathcal{H})+\mathrm{E}^{g}(\xi \mid \mathcal{H}) \quad \text { a.s. }
$$

Proof. Since $X-\xi$ is integrable, $\mathrm{E}(X-\xi \mid \mathcal{H})=\mathrm{E}^{g}(X-\xi \mid \mathcal{H})$ by Lemma 3.13. Then

$$
\mathrm{E}^{g}(\eta \mid \mathcal{H})=\mathrm{E}^{g}(\eta-\xi \mid \mathcal{H})+\mathrm{E}^{g}(\xi \mid \mathcal{H}) \in \mathrm{E}^{g}(X-\xi \mid \mathcal{H})+\mathrm{E}^{g}(\xi \mid \mathcal{H}) \quad \text { a.s. }
$$

for all $\eta \in \mathcal{L}_{\mathcal{H}}^{1}(X, \mathcal{F})$. Therefore, $\mathrm{E}^{g}(X \mid \mathcal{H}) \subset \mathrm{E}(X-\xi \mid \mathcal{H})+\mathrm{E}^{g}(\xi \mid \mathcal{H})$ a.s. Since $\mathrm{E}^{g}(X-\xi \mid \mathcal{H}) \subset \mathrm{E}^{g}(X \mid \mathcal{H})-\mathrm{E}^{g}(\xi \mid \mathcal{H})$, the reverse inclusion follows.

Lemma 3.15. Let $X$ be a random closed set that contains 0 a.s. and let $X^{n}=X \cap n B, n \geqslant 1$. Then

$$
\mathrm{E}(X \mid \mathcal{H})=\operatorname{cl} \bigcup_{n \geqslant 1} \mathrm{E}\left(X^{n} \mid \mathcal{H}\right) \quad \text { a.s. }
$$

Proof. Denote the right-hand side by $Y$. Note that $Y \subset \mathrm{E}(X \mid \mathcal{H})$. To confirm the reverse inclusion, let $\xi=\mathrm{E}(\eta \mid \mathcal{H})$ for $\eta \in \mathcal{L}^{1}(X, \mathcal{F})$. Then $\xi$ is the limit of $\mathrm{E}\left(\eta_{n} \mid \mathcal{H}\right)$ in $\mathcal{L}^{1}$, where $\eta_{n}=\eta \mathbf{1}_{|\eta| \leqslant n} \in \mathcal{L}^{1}\left(X^{n}, \mathcal{F}\right)$. Since $\mathrm{E}\left(\eta_{n} \mid \mathcal{H}\right) \in \mathrm{E}\left(X^{n} \mid \mathcal{H}\right)$ a.s., $\xi \in Y$ a.s. and the conclusion follows. 
Proposition 3.16. If $X$ is a random closed convex set and $\mathcal{L}^{0}(X, \mathcal{H}) \neq$ $\varnothing$, then

$$
\mathbf{m}(X \mid \mathcal{H}) \subset \mathrm{E}^{g}(X \mid \mathcal{H}) \subset \mathbf{M}(X \mid \mathcal{H}) \text { a.s. }
$$

Proof. Since $\mathcal{L}^{0}(X, \mathcal{H}) \neq \varnothing$, we may replace $X$ by $X-\xi$ for $\xi \in$ $\mathcal{L}^{0}(X, \mathcal{H})$ and so assume that $0 \in X$ a.s. and $\mathrm{E}^{g}(X \mid \mathcal{H})=\mathrm{E}(X \mid \mathcal{H})$. For $\gamma \in \mathcal{L}^{0}(\mathbf{m}(X \mid \mathcal{H}), \mathcal{H}), \gamma=\mathrm{E}^{g}(\gamma \mid \mathcal{H}) \in \mathrm{E}^{g}(X \mid \mathcal{H})$ a.s. The second inclusion follows from Proposition 3.10 and Lemma 3.15.

Corollary 3.17. If $X$ is an $\mathcal{H}$-measurable a.s. non-empty random closed convex set, then $\mathrm{E}^{g}(X \mid \mathcal{H})=X$ a.s.

While the conditional core is a superlinear set-valued functional of random closed sets by (3.1), the conditional convex hull satisfies

$$
\mathbf{M}(X+Y \mid \mathcal{H}) \subset \mathbf{M}(X \mid \mathcal{H})+\mathbf{M}(Y \mid \mathcal{H})
$$

and so is sublinear. The following result can be viewed as an analogue of the representation of superlinear and sublinear functions as suprema and infima of linear functions. Denote by $\mathcal{Q}$ the family of all probability measures $Q$ absolutely continuous with respect to $P$.

Proposition 3.18. Assume that $\mathcal{L}^{\infty}(X, \mathcal{H}) \neq \varnothing$.

(i) $\mathbf{M}(X \mid \mathcal{H})$ is the smallest $\mathcal{H}$-measurable random closed convex set a.s. containing $\mathrm{E}_{\mathrm{Q}}(X \mid \mathcal{H})$ for all $\mathrm{Q} \in \mathcal{Q}$.

(ii) If $X$ is a.s. bounded and convex, then $\mathbf{m}(X \mid \mathcal{H})$ is the largest $\mathcal{H}$ measurable random closed set contained a.s. in $\mathrm{E}_{Q}(X \mid \mathcal{H})$ for all $\mathrm{Q} \in \mathcal{Q}$.

Proof. (i) Assume that $X$ contains the origin almost surely. Let $Z$ be any $\mathcal{H}$-measurable random closed convex set containing $\mathrm{E}_{\mathrm{Q}}(X \mid \mathcal{H})$ for all $\mathrm{Q} \in \mathcal{Q}$. If $X$ is a.s. bounded, then $\mathrm{E}_{\mathrm{Q}}(X \mid \mathcal{H}) \subset Z$ a.s. implies $h_{\mathbf{M}(X \mid \mathcal{H})}(u) \leqslant h_{Z}(u)$ for all $u \in \mathbb{Q}^{d}$ a.s. and then for all $u \in \mathbb{R}^{d}$ by the continuity of the support function. The result follows from Theorem A.2 taking into account that the support function of $X$ is a.s. non-negative and the second part of Proposition 3.10.

If $X$ is not necessarily bounded, let $X^{n}=X \cap n B$ and $Z^{n}=Z \cap$ $n B$ for $n \geqslant 1$. Since $\mathrm{E}_{\mathrm{Q}}\left(X^{n} \mid \mathcal{H}\right) \subset Z^{n}$ a.s. for all $\mathrm{Q} \in \mathcal{Q}$, we have $\mathbf{M}\left(X^{n} \mid \mathcal{H}\right) \subset Z^{n}$ a.s. Therefore, $\mathbf{M}(X \mid \mathcal{H})=\operatorname{cl} \bigcup_{n} \mathbf{M}\left(X^{n} \mid \mathcal{H}\right) \subset Z$ a.s.

More generally, suppose there exists $\gamma \in \mathcal{L}^{\infty}(X, \mathcal{H})$. Then $X^{\prime}=X-\gamma$ a.s. contains the origin, $\mathbf{M}\left(X^{\prime} \mid \mathcal{H}\right)=\mathbf{M}(X \mid \mathcal{H})-\gamma$, and $\mathrm{E}_{Q}\left(X^{\prime} \mid \mathcal{H}\right)=$ $\mathrm{E}_{\mathrm{Q}}(X \mid \mathcal{H})-\gamma$, so that the claim still holds.

(ii) Consider an $\mathcal{H}$-measurable random convex closed set $Z$ such that $Z \subset \mathrm{E}_{\mathrm{Q}}(X \mid \mathcal{H})$ a.s. for all $\mathrm{Q} \in \mathcal{Q}$. Then, $h_{Z}(u) \leqslant \operatorname{essinf}_{\mathcal{H}} h_{X}(u)$ by Theorem A.2, for all $u \in \mathbb{Q}^{d}$ a.s. By Proposition 3.8, $Z \subset \mathbf{m}(X \mid \mathcal{H})$ if $X$ is bounded. 
3.4. Random cones. If $K$ is a convex cone, then its positive dual cone is defined by

$$
K^{*}=\left\{x \in \mathbb{R}^{d}:\langle x, y\rangle \geqslant 0 \forall y \in K\right\} .
$$

Proposition 3.19. Let $K$ be a random convex closed cone in $\mathbb{R}^{d}$. Then both $\mathbf{m}(K \mid \mathcal{H})$ and $\mathbf{M}(K \mid \mathcal{H})$ are closed convex cones, $\mathbf{m}(K \mid \mathcal{H})=$ $\mathbf{M}\left(K^{*} \mid \mathcal{H}\right)^{*}$, and $\mathrm{E}(K \mid \mathcal{H})=\mathbf{M}(K \mid \mathcal{H})$ a.s.

Proof. The conical properties of the core and the convex hull are obvious. Since $\mathbf{m}(K \mid \mathcal{H}) \subset K$,

$$
K^{*} \subset \mathbf{M}\left(K^{*} \mid \mathcal{H}\right) \subset \mathbf{m}(K \mid \mathcal{H})^{*}
$$

in view of the definition of $\mathbf{M}\left(K^{*} \mid \mathcal{H}\right)$. Therefore, $\mathbf{m}(K \mid \mathcal{H}) \subset$ $\mathbf{M}\left(K^{*} \mid \mathcal{H}\right)^{*}$. The opposite inclusion follows from $\mathbf{M}\left(K^{*} \mid \mathcal{H}\right)^{*} \subset$ $\mathbf{m}(K \mid \mathcal{H}) \subset K$ by the definition of the $\mathcal{H}$-core.

For the last statement, it suffices to show that $\mathrm{E}(K \mid \mathcal{H})^{*}=\mathbf{M}(K \mid \mathcal{H})^{*}$, equivalently $\mathrm{E}(K \mid \mathcal{H})^{*}=\mathbf{m}\left(K^{*} \mid \mathcal{H}\right)$. Observe that $\gamma \in \mathcal{L}^{1}\left(\mathrm{E}(K \mid \mathcal{H})^{*}, \mathcal{H}\right)$ if and only if $\mathrm{E}\langle\mathrm{E}(k \mid \mathcal{H}), \gamma\rangle \geqslant 0$ for all $k \in \mathcal{L}^{1}(K, \mathcal{F})$, i.e. $\mathrm{E}\langle k, \gamma\rangle \geqslant 0$ for all $k \in \mathcal{L}^{1}(K, \mathcal{F})$, or equivalently $\gamma \in K^{*}$ a.s., i.e. $\gamma \in \mathbf{m}\left(K^{*} \mid \mathcal{H}\right)$.

Example 3.20 (Random cone in $\mathbb{R}^{2}$ ). Consider $Y=\left[S^{b}, S^{a}\right]$ for $S^{a}, S^{b} \in$ $\mathcal{L}^{0}\left(\mathbb{R}_{+}, \mathcal{F}\right)$ with $S^{b} \leqslant S^{a}$ a.s. and the cone $K=C(Y)$ generated by $\left(S^{a} e_{1}-e_{2}\right)$ and $\left(-S^{b} e_{1}+e_{2}\right)$, where $e_{1}=(1,0)$ and $e_{2}=(0,1)$ are the standard basis vectors in $\mathbb{R}^{2}$. In finance, it models solvent positions for the bid-ask spread $\left[S^{b}, S^{a}\right]$. Note that $K$ is the dual to the cone $\cup_{t \geqslant 0} t(1 \times Y)$. By Proposition 3.19, $\mathbf{m}(K \mid \mathcal{H})=C(\mathbf{M}(Y \mid \mathcal{H}))$ and $\mathbf{M}(K \mid \mathcal{H})=\mathrm{E}(K \mid \mathcal{H})=C(\mathbf{m}(Y \mid \mathcal{H}))$.

\section{DynamiC ACCEPTANCE SETS AND SELECTION RISK MEASURES}

4.1. Definition and main properties. Let $\left(\Omega,\left(\mathcal{F}_{t}\right)_{t=0, \ldots, T}, \mathrm{P}\right)$ be a stochastic basis on a complete probability space such that $\mathcal{F}_{0}$ is the trivial $\sigma$-algebra. Furthermore, let $\overline{\mathcal{L}}_{\mathcal{H}}^{p}\left(\mathbb{R}^{d}, \mathcal{F}\right)$ denote the family of random vectors $\xi$ that can be decomposed as $\xi=\xi^{\prime}+\xi^{\prime \prime}$, where $\xi^{\prime} \in$ $\mathcal{L}_{\mathcal{H}}^{p}\left(\mathbb{R}^{d}, \mathcal{F}\right)$ and $\xi^{\prime \prime} \geqslant 0$ a.s., see Appendix B. In the following, we mostly endow random vectors with a subscript that indicates the $\sigma$-algebras they are measurable with respect to.

Definition 4.1. Discrete time $\mathcal{L}^{p}$-dynamic convex acceptance sets are the family $\left\{\mathcal{C}_{t, s}, 0 \leqslant t \leqslant s \leqslant T\right\}$, such that $\mathcal{C}_{t, s} \subset \overline{\mathcal{L}}_{\mathcal{F}_{t}}^{p}\left(\mathbb{R}^{d}, \mathcal{F}_{s}\right)$ and the following properties hold for all $0 \leqslant t \leqslant s \leqslant T$.

(i) Normalisation: $\mathcal{C}_{t, s} \supset \mathcal{L}^{0}\left(\mathbb{R}_{+}^{d}, \mathcal{F}_{s}\right)$, and $\mathcal{C}_{t, s} \cap \mathcal{L}^{0}\left(\mathbb{R}_{-}^{d}, \mathcal{F}_{s}\right)=\{0\}$.

(ii) Integrability:

$$
\mathcal{C}_{t, s}=\left(\mathcal{C}_{t, s} \cap \mathcal{L}_{\mathcal{F}_{t}}^{p}\left(\mathbb{R}^{d}, \mathcal{F}_{s}\right)\right)+\mathcal{L}^{0}\left(\mathbb{R}_{+}^{d}, \mathcal{F}_{s}\right)
$$


(iii) Closedness: $\mathcal{C}_{t, s} \cap \mathcal{L}_{\mathcal{F}_{t}}^{p}\left(\mathbb{R}^{d}, \mathcal{F}_{T}\right)$ is closed with respect to the $\|\cdot\|_{p, \mathcal{H}^{-}}$ norm if $p \in[1, \infty)$ and with respect to the $\mathcal{F}_{t}$-bounded convergence in probability if $p=\infty$.

(iv) Conditional convexity: for all $\alpha_{t} \in \mathcal{L}^{0}\left([0,1], \mathcal{F}_{t}\right)$, and $\eta_{s}^{\prime}, \eta_{s}^{\prime \prime} \in \mathcal{C}_{t, s}$,

$$
\alpha_{t} \eta_{s}^{\prime}+\left(1-\alpha_{t}\right) \eta_{s}^{\prime \prime} \in \mathcal{C}_{t, s}
$$

(v) Weak time consistency: $\mathcal{C}_{t, s} \cap \mathcal{L}^{0}\left(\mathbb{R}^{d}, \mathcal{F}_{u}\right)=\mathcal{C}_{t, u}$ for all $0 \leqslant t \leqslant$ $u \leqslant s \leqslant T$.

(vi) Compensation: if $\xi_{s} \in \mathcal{L}_{\mathcal{F}_{t}}^{p}\left(\mathbb{R}^{d}, \mathcal{F}_{s}\right)$, then $\left(\xi_{s}+\mathcal{C}_{t, s}\right) \cap \mathcal{L}^{0}\left(\mathbb{R}^{d}, \mathcal{F}_{t}\right) \neq$ $\varnothing$, and $\left(\xi_{s}+\mathcal{C}_{t, s}\right) \cap \mathcal{L}^{p}\left(\mathbb{R}^{d}, \mathcal{F}_{t}\right) \neq \varnothing$ if $\xi_{s} \in \mathcal{L}^{p}\left(\mathbb{R}^{d}, \mathcal{F}_{s}\right)$.

It is convenient to let $\mathcal{C}_{-1,0}=\mathcal{L}^{0}\left(\mathbb{R}_{+}^{d}, \mathcal{F}_{0}\right)$. The integrability property implies that $\mathcal{C}_{t, s}$ is an upper set, that is $\eta_{s} \in \mathcal{C}_{t, s}$ and $\eta_{s} \leqslant \eta_{s}^{\prime}$ a.s. yield $\eta_{s}^{\prime} \in \mathcal{C}_{t, s}$. In many cases, $\mathcal{C}_{t, s}$ is constructed as the Cartesian product of acceptance sets in dimension one, see Remark 4.6.

Example 4.2 (Static convex risk measures). Consider the one-period setting in one dimension with $t=0,1$. If $r$ is a convex $\mathcal{L}^{p}$-risk measure with $p \in[1, \infty)$, then its acceptance set $\mathcal{C}_{0,1} \cap \mathcal{L}^{p}\left(\mathbb{R}, \mathcal{F}_{1}\right)$ is the family of $\eta_{1} \in \mathcal{L}^{p}\left(\mathbb{R}, \mathcal{F}_{1}\right)$ such that $r\left(\eta_{1}\right) \leqslant 0$. It is known that the Fatou property of $r$ is equivalent to the closedness of the acceptance set, see [18, Lemma 3.2]. The conditional convexity property of the acceptance set is equivalent to the convexity property of the risk measure. The compensation property corresponds to the finiteness of $r$.

Lemma 4.3. The sets $\overline{\mathcal{L}}_{\mathcal{F}_{t}}^{p}\left(\mathbb{R}^{d}, \mathcal{F}_{s}\right)$ and $\mathcal{C}_{t, s}$ are infinitely $\mathcal{F}_{t}$-decomposable for all $0 \leqslant t \leqslant s \leqslant T$.

Proof. Let $\xi_{s}^{i} \in \mathcal{L}_{\mathcal{F}_{t}}^{p}\left(\mathbb{R}^{d}, \mathcal{F}_{s}\right), i \geqslant 1$, and let $\left(B_{t}^{i}\right)_{i \geqslant 1}$ be an $\mathcal{F}_{t}$-measurable partition. Note that $\xi_{s}=\sum_{i=1}^{\infty} \mathbf{1}_{B_{t}^{i}} \xi_{s}^{i}$ satisfies the equality

$$
\mathrm{E}\left(\left\|\xi_{s}\right\|^{p} \mid \mathcal{F}_{t}\right)=\sum_{i=1}^{\infty} \mathrm{E}\left(\left\|\xi_{s}^{i}\right\|^{p} \mid \mathcal{F}_{t}\right) \mathbf{1}_{B_{t}^{i}} .
$$

Then $\mathrm{E}\left(\left\|\xi_{s}\right\|^{p} \mid \mathcal{F}_{t}\right)<\infty$ a.s., that is $\xi_{s} \in \mathcal{L}_{\mathcal{F}_{t}}^{p}\left(\mathbb{R}^{d}, \mathcal{F}_{s}\right)$ by Theorem B.2.

The conditional convexity property yields that $\mathcal{C}_{t, s} \cap \mathcal{L}_{\mathcal{F}_{t}}^{p}\left(\mathbb{R}^{d}, \mathcal{F}_{s}\right)$ is $\mathcal{F}_{t^{-}}$decomposable. In particular, if $\eta_{s}^{i} \in \mathcal{C}_{t, s} \cap \mathcal{L}_{\mathcal{F}_{t}}^{p}\left(\mathbb{R}^{d}, \mathcal{F}_{s}\right), i \geqslant 1$, then

$$
\bar{\eta}_{s}^{n}=\sum_{i=1}^{n} \mathbf{1}_{B_{t}^{i}} \eta_{s}^{i}+\eta_{s}^{1} \mathbf{1}_{\Omega \backslash \cup_{i=1}^{n} B_{t}^{i}} \in \mathcal{C}_{t, s} .
$$

Since $\bar{\eta}_{s}^{n} \rightarrow \bar{\eta}_{s}=\sum_{i=1}^{\infty} \mathbf{1}_{B_{t}^{i}} \eta_{s}^{i}$ in the $\|\cdot\|_{p, \mathcal{F}_{t}}$-norm if $p \in[1, \infty)$ and $\mathcal{F}_{t}$-boundedly a.s. if $p=\infty$, we have $\bar{\eta}_{s} \in \mathcal{C}_{t, s} \cap \mathcal{L}_{\mathcal{F}_{t}}^{p}\left(\mathbb{R}^{d}, \mathcal{F}_{s}\right)$. By the integrability property, $\mathcal{C}_{t, s}$ is also infinitely decomposable. 
Definition 4.4. The family of acceptance sets is called

(i) coherent if $\alpha_{t} \eta_{s} \in \mathcal{C}_{t, s}$ for all $t \leqslant s, \alpha_{t} \in \mathcal{L}^{0}\left([0, \infty), \mathcal{F}_{t}\right)$, and $\eta_{s} \in \mathcal{C}_{t, s}$

(ii) dilatation monotonic if

$$
\mathcal{C}_{t, s} \supset\left\{\mathrm{E}^{g}\left(\eta_{T} \mid \mathcal{F}_{s}\right): \eta_{T} \in \mathcal{C}_{t, T} \cap \mathcal{L}_{\mathcal{F}_{t}}^{1}\left(\mathbb{R}^{d}, \mathcal{F}_{T}\right)\right\}, \quad 0 \leqslant t \leqslant s \leqslant T .
$$

The dilatation monotonicity yields that $\mathrm{E}^{g}\left(\eta_{s} \mid \mathcal{F}_{t}\right) \in \mathcal{L}^{0}\left(\mathbb{R}_{+}^{d}, \mathcal{F}_{t}\right)$ for all $t \leqslant s$ and $\eta_{s} \in \mathcal{C}_{t, s} \cap \mathcal{L}_{\mathcal{F}_{t}}^{1}\left(\mathbb{R}^{d}, \mathcal{F}_{s}\right)$. It is well known that static law invariant convex risk measures on non-atomic probability spaces are dilatation monotonic, see e.g. [4] and [10]. In an accompanying paper, we show that this is also the case under the following condition.

Definition 4.5. The acceptance sets are said to be continuous from below if, for all $t \leqslant s$, and any sequence $\xi_{s}^{n} \in \mathcal{L}_{\mathcal{F}_{t}}^{p}\left(\mathbb{R}^{d}, \mathcal{F}_{s}\right), n \geqslant 1$, such that

$$
\xi_{s}^{n} \uparrow \xi_{s} \in \mathcal{C}_{t, s} \cap \mathcal{L}_{\mathcal{F}_{t}}^{p}\left(\mathbb{R}^{d}, \mathcal{F}_{s}\right) \quad \text { a.s. as } n \rightarrow \infty
$$

and $\left\|\xi_{s}^{n}-\xi_{s}\right\|_{p, \mathcal{F}_{t}} \rightarrow 0$, there exists a sequence $\gamma_{t}^{n} \in \mathcal{L}^{0}\left(\mathbb{R}_{+}^{d}, \mathcal{F}_{t}\right), n \geqslant 1$, such that $\gamma_{t}^{n}+\xi_{s}^{n} \in \mathcal{C}_{t, s}, \gamma_{t}^{n} \downarrow 0$ a.s., and $\gamma_{t}^{1} \leqslant-\operatorname{essinf}_{\mathcal{F}_{t}} \xi_{s}^{1}$ in case ess $\inf _{\mathcal{F}_{t}} \xi_{s}^{1}$ is a.s. finite.

Remark 4.6. The acceptance sets can be defined using a convex dynamic $\mathcal{L}^{p}$-risk measure $\left(r_{t}\right)_{t=0, \ldots, T}$, so that

$$
\xi_{s}=\left(\xi_{s}^{1}, \ldots, \xi_{s}^{d}\right) \in \mathcal{C}_{t, s} \cap \mathcal{L}_{\mathcal{F}_{t}}^{p}\left(\mathbb{R}^{d}, \mathcal{F}_{s}\right)
$$

if and only if $r_{t}\left(\xi_{s}^{i}\right) \leqslant 0$ for all $i=1, \ldots, d$. The continuity from below property holds if $r_{t}$ is lower semicontinuous in the $\|\cdot\|_{p, \mathcal{F}_{t}}$-norm and continuous from below, which is the case if $p \in[1, \infty), r_{t}$ is convex and a.s. finite, see [26, Th. 4.1.4].

4.2. Dynamic selection risk measures. Let $\Xi$ be an upper subset of $\mathcal{L}^{0}\left(\mathbb{R}^{d}, \mathcal{F}_{T}\right)$, that is with each $\xi_{T} \in \Xi$, the family $\Xi$ also contains all $\xi_{T}^{\prime} \in \mathcal{L}^{0}\left(\xi+\mathbb{R}_{+}^{d}, \mathcal{F}_{T}\right)$. The most important example of such family is $\mathcal{L}^{0}\left(X, \mathcal{F}_{T}\right)$ for an $\mathcal{F}_{T}$-measurable upper random set $X$ in $\mathbb{R}^{d}$, that is $X+\mathbb{R}_{+}^{d} \subset X$ a.s. If $X$ is also closed, then its centrally symmetric version $(-X)$ is a set-valued portfolio in the terminology of [22].

Definition 4.7. Let $\Xi \subset \mathcal{L}^{0}\left(\mathbb{R}^{d}, \mathcal{F}_{T}\right)$ be an upper set. For $t \leqslant s \leqslant T$, $\mathrm{R}_{t, s}^{0}(\Xi)$ denotes the set of all $\gamma \in \mathcal{L}^{0}\left(\mathbb{R}^{d}, \mathcal{F}_{t}\right)$ such that $\gamma-\xi \in \mathcal{C}_{t, s}$ for some $\xi \in \Xi$. The dynamic selection risk measure $\mathrm{R}_{t, s}(\Xi)$ is the $\mathcal{L}^{0}$ closure of $\mathrm{R}_{t, s}^{0}(\Xi)$. If $\Xi=\mathcal{L}^{0}\left(X, \mathcal{F}_{T}\right)$ for an upper random set $X$, we write $\mathrm{R}_{t, s}^{0}(X)$ and $\mathrm{R}_{t, s}(X)$ instead of $\mathrm{R}_{t, s}^{0}(\Xi)$ and $\mathrm{R}_{t, s}(\Xi)$.

Equivalently,

$$
\mathrm{R}_{t, s}^{0}(\Xi)=\left(\Xi+\mathcal{C}_{t, s}\right) \cap \mathcal{L}^{0}\left(\mathbb{R}^{d}, \mathcal{F}_{t}\right)
$$


Note that $\mathrm{R}_{T, T}^{0}(\Xi)=\Xi$, and $\mathrm{R}_{t, u}^{0}(\Xi) \subset \mathrm{R}_{t, s}^{0}(\Xi)$ for all $0 \leqslant t \leqslant u \leqslant s \leqslant$ $T$. If only portfolios from a random set $M_{t}$ are allowed for compensation at time $t$, like it is the case in [7], it is possible to modify the definition by considering the intersection of $\left(\Xi+\mathcal{C}_{t, s}\right)$ with $\mathcal{L}^{0}\left(M_{t}, \mathcal{F}_{t}\right)$. In difference with [7], we include the solvency sets in the arguments of the risk measures. This makes it possible to work with positions dependent on solvency sets (e.g. exchange rates) and in many cases ensures the law invariance property. In the static setting, the set $R_{0,1}\left(\xi+\mathbb{R}_{+}^{d}\right)$ is called the regulator risk measure and $\mathrm{R}_{0,1}\left(\xi+K_{1}\right)$ its market extension, see [13].

The compensation property of acceptance sets guarantees that $\mathrm{R}_{t, s}^{0}(\Xi)$ is not empty if $\Xi \cap \mathcal{L}_{\mathcal{F}_{t}}^{p}\left(\mathbb{R}^{d}, \mathcal{F}_{s}\right) \neq \varnothing$. The empty selection risk measure corresponds to completely unacceptable positions. This is the case, for instance, if $\Xi \cap \mathcal{L}^{0}\left(\mathbb{R}^{d}, \mathcal{F}_{s}\right)=\varnothing$ or if the random set set $X$ is empty with a positive probability. For each $s \geqslant t$, Lemma 4.3 yields that $\mathrm{R}_{t, s}^{0}(\Xi)$ and $\mathrm{R}_{t, s}(\Xi)$ are infinitely $\mathcal{F}_{t}$-decomposable if $\Xi$ is infinitely $\mathcal{F}_{t}$-decomposable. The selection risk measure is conditionally convex, that is

$$
\mathrm{R}_{t, s}^{0}\left(\alpha_{t} X+\left(1-\alpha_{t}\right) Y\right) \supset \alpha_{t} \mathrm{R}_{t, s}^{0}(X)+\left(1-\alpha_{t}\right) \mathrm{R}_{t, s}^{0}(Y)
$$

for all $\alpha_{t} \in \mathcal{L}^{0}\left([0,1], \mathcal{F}_{t}\right)$, and the same holds for the closures. The family $\Xi$ is said to be acceptable for the time horizon $s$ if $0 \in \mathrm{R}_{t, s}^{0}(\Xi)$, that is $-\Xi$ contains an element from $\mathcal{C}_{t, s}$.

Lemma 4.8. Let $X$ be an $\mathcal{F}_{T}$-measurable random upper set.

(i) $\mathrm{R}_{t, s}(X)$ is the family of selections of an $\mathcal{F}_{t}$-measurable upper random closed set in $\mathbb{R}^{d}$ that is also denoted by $\mathrm{R}_{t, s}(X)$.

(ii) If $X$ is a.s. convex, then $\mathrm{R}_{t, s}^{0}(X)$ is a.s. convex. If $X$ is a cone and the acceptance sets are coherent, then $\mathrm{R}_{t, s}^{0}(X)$ is a cone.

(iii) If $\mathbf{m}\left(X \mid \mathcal{F}_{t}\right)$ exists, then $\mathbf{m}\left(X \mid \mathcal{F}_{t}\right) \subset \mathrm{R}_{t, s}^{0}(X)$.

Proof. (i) By Lemma 4.3, $\mathrm{R}_{t, s}(X)$ is the closure of an $\mathcal{F}_{t}$-decomposable family, and so Theorem 2.3 applies.

(ii) If $\gamma_{t}^{1}, \gamma_{t}^{2} \in \mathrm{R}_{t, s}^{0}(X)$, then $\gamma_{t}^{i}-\xi_{s}^{i} \in \mathcal{C}_{t, s}, i=1,2$, for $\xi_{s}^{1}, \xi_{s}^{2} \in$ $\mathcal{L}^{0}\left(X, \mathcal{F}_{s}\right)$. For any $t \in(0,1)$, the conditional convexity property yields that $t \gamma_{t}^{1}+(1-t) \gamma_{t}^{2}-\xi_{s} \in \mathcal{C}_{t, s}$ with $\xi_{s}=t \xi_{s}^{1}+(1-t) \xi_{s}^{2} \in \mathcal{L}^{0}\left(X, \mathcal{F}_{s}\right)$. The conical property is trivial.

(iii) We have $\mathrm{R}_{t, s}(X) \supset \mathcal{L}^{0}\left(X, \mathcal{F}_{t}\right)$ by $(4.2)$.

Remark 4.9. If the acceptance sets are dilatation monotonic and $X$ is a.s. convex, then $\mathrm{R}_{t, s}(X) \subset \mathrm{E}^{g}\left(X \mid \mathcal{F}_{t}\right)$ for all $t \leqslant s$.

Example 4.10. If $X=\left[\xi_{s}, \infty\right)$ in $\mathbb{R}$ for $\xi_{s} \in \mathcal{L}_{\mathcal{F}_{t}}^{p}\left(\mathbb{R}, \mathcal{F}_{s}\right)$, then $\mathrm{R}_{t, s}^{0}(X)=$ $\mathrm{R}_{t, s}(X)=\left[r_{t}\left(-\xi_{s}\right), \infty\right)$ for a dynamic risk measure $r_{t}$ on $\mathcal{L}_{\mathcal{F}_{t}}^{p}\left(\mathbb{R}, \mathcal{F}_{s}\right)$. 
Example 4.11. The entropic risk measure from [8, Sec. 3.2] can be also used to define the entropic selection risk measure. It corresponds to the choice $p=\infty$ and

$$
\mathcal{C}_{t, s}=\left\{\xi_{s} \in \mathcal{L}_{\mathcal{F}_{t}}^{\infty}\left(\mathbb{R}_{+}^{d}, \mathcal{F}_{s}\right): \mathrm{E}\left(e^{-\xi_{s}} \mid \mathcal{F}_{t}\right) \leqslant \mathbf{1}\right\}+\mathcal{L}^{0}\left(\mathbb{R}_{+}^{d}, \mathcal{F}_{s}\right) .
$$

\section{Hedging to aCCePtability}

Let $\left(K_{t}\right)_{t=0, \ldots, T}$ be a sequence of random closed convex sets, such that $K_{t} \cap \mathbb{R}_{-}^{d}=\{0\}, K_{t}$ is an upper set, and $K_{t}$ is $\mathcal{F}_{t}$-measurable for all $t$. The set $K_{t}$ is interpreted as the set of all solvent positions at time $t$ expressed in physical units and is called a solvency set, see [17]. If the solvency sets are cones, this model is well studied under the name of Kabanov's model; it describes the market subject to proportional transaction costs, see $[17,25]$. If the solvency sets are cones and the acceptance sets are coherent, we talk about the coherent conical setting.

Let $K_{t}^{0}$ be the largest $\mathcal{F}_{t}$-measurable linear subspace contained in $K_{t}$, that is

$$
K_{t}^{0}=\bigcap_{c \neq 0} c K_{t}=\bigcap_{c \in \mathbb{Q} \backslash\{0\}} c K_{t} .
$$

The solvency sets are said to be proper if $K_{t}^{0}=\{0\}$ and strictly proper if $\tilde{K}_{t}=K_{t} \cap\left(-K_{t}\right)=\{0\}$ for all $t=0, \ldots, T$. If $K_{t}$ is a cone, then $\tilde{K}_{t}=K_{t}^{0}$, while in general $K_{t}^{0}=\cap_{c \neq 0} c \tilde{K}_{t} \subset \tilde{K}_{t}$. Since $\tilde{K}_{t}$ is convex and centrally symmetric, $K_{t}$ is proper if and only if $\tilde{K}_{t}$ is bounded.

Definition 5.1. An $\mathcal{F}_{t}$-adapted sequence $\left(V_{t}\right)_{t=0, \ldots, T}$ is called an acceptable portfolio process if

$$
V_{t-1}-V_{t} \in \mathcal{L}^{0}\left(K_{t}, \mathcal{F}_{t}\right)+\mathcal{C}_{t-1, t}, \quad t=1, \ldots, T .
$$

By the definition of the selection risk measure, (5.1) is equivalent to

$$
V_{t-1} \in \mathrm{R}_{t-1, t}^{0}\left(V_{t}+K_{t}\right), \quad t=1, \ldots, T .
$$

Thus, paying transaction costs, it is possible to transform $V_{t-1}-V_{t}$ into an acceptable to the horizon $t$ position. If the dilatation monotonicity property is assumed, the acceptability can be equivalently defined for later horizons.

The initial endowment for an acceptable portfolio process is any $V_{0-} \in \mathcal{L}^{0}\left(V_{0}+K_{0}, \mathcal{F}_{0}\right)$, so that it is possible to convert $V_{0-}$ into $V_{0}$ paying the transaction costs. The endowment $V_{t-}$ at time $t$ is defined similarly.

The family of attainable positions at time $s>t$ is the family of random vectors that may be obtained as $V_{s}$ for acceptable portfolio 
processes starting from zero investment at time $t$. By (5.1), the family of attainable positions is given by

$$
\mathrm{A}_{t, s}=\sum_{u=t}^{s} \mathcal{L}^{0}\left(-K_{u}, \mathcal{F}_{u}\right)-\sum_{u=t}^{s-1} \mathcal{C}_{u, u+1}
$$

Let $\overline{\mathrm{A}}_{t, s}^{p}$ denote the closure of $\mathrm{A}_{t, s}^{p}=\mathrm{A}_{t, s} \cap \mathcal{L}^{p}\left(\mathbb{R}^{d}, \mathcal{F}_{T}\right)$ in $\mathcal{L}^{p}$ for $p<\infty$ and in the $\mathcal{F}_{t^{-}}$bounded convergence in probability for $p=\infty$.

Let $\xi \in \mathcal{L}^{0}\left(\mathbb{R}^{d}, \mathcal{F}_{T}\right)$ be a terminal claim (or payoff). The hedging to acceptability aims to come up with an acceptable portfolio process $\left(V_{t}\right)_{t=0, \ldots, T}$ that guarantees paying $\xi$ in the sense that the terminal wealth $V_{T}$ belongs to $\Xi_{T}^{\xi}=\mathcal{L}^{0}\left(X_{T}^{\xi}, \mathcal{F}_{T}\right)$, the family of selections of the random closed set $X_{T}^{\xi}=\xi+K_{T}$. Define recursively

$$
\Xi_{t}^{\xi}=\mathcal{L}^{0}\left(K_{t}, \mathcal{F}_{t}\right)+\mathrm{R}_{t, t+1}^{0}\left(\Xi_{t+1}^{\xi}\right), \quad t=T-1, \ldots, 0 .
$$

If $\xi=\xi^{\prime}-\xi^{\prime \prime}$ for $\xi^{\prime} \in \mathcal{L}^{p}\left(\mathbb{R}^{d}, \mathcal{F}_{T}\right)$ and $\xi^{\prime \prime} \in \mathcal{L}^{0}\left(\mathbb{R}_{+}^{d}, \mathcal{F}_{T}\right)$, the compensation property of acceptance sets ensures that $\Xi_{t}^{\xi}$ is not empty for all $t$. The family $\Xi_{t}^{\xi}$ describes the superhedging prices for $\xi$ and so may serve as a conditionally convex risk measure of $\xi$.

In order to handle the asymptotic setting, let $\hat{\Xi}_{T}^{\xi}=\Xi_{T}^{\xi}$, and

$$
\hat{\Xi}_{t}^{\xi}=\mathcal{L}^{0}\left(K_{t}, \mathcal{F}_{t}\right)+\mathrm{R}_{t, t+1}\left(\Xi_{t+1}^{\xi}\right), \quad t=T-1, \ldots, 0 .
$$

Note that $\Xi_{t}^{\xi} \subset \hat{\Xi}_{t}^{\xi} \subset \operatorname{cl}_{0}\left(\Xi_{t}^{\xi}\right)$, whence $\operatorname{cl}_{0}\left(\hat{\Xi}_{t}^{\xi}\right)=\operatorname{cl}_{0}\left(\Xi_{t}^{\xi}\right)$ for all $t$.

Lemma 5.2. (i) The families $\mathrm{R}_{t, s}^{0}\left(\Xi_{s}^{\xi}\right)$ and $\mathrm{R}_{t, s}\left(\Xi_{s}^{\xi}\right)$ are convex and infinitely $\mathcal{F}_{t}$-decomposable for all $0 \leqslant t \leqslant s \leqslant T$.

(ii) For each $t \leqslant T$, there exists a (possibly non-closed) random set $X_{t}^{\xi}$ such that $\hat{\Xi}_{t}^{\xi}=\mathcal{L}^{0}\left(X_{t}^{\xi}, \mathcal{F}_{t}\right)$.

(iii) For any $t \leqslant T$, the family of all initial endowments $V_{t-}$ at time $t$ allowing to start an acceptable portfolio process $\left(V_{s}\right)_{t \leqslant s \leqslant T}$ such that $V_{T} \in \mathcal{L}^{0}\left(\xi+K_{T}, \mathcal{F}_{T}\right)$ a.s. coincides with $\Xi_{t}^{\xi}$ and

$$
\Xi_{t}^{\xi}=\left(-\mathrm{A}_{t, T}+\xi\right) \cap \mathcal{L}^{0}\left(\mathbb{R}^{d}, \mathcal{F}_{t}\right) .
$$

(iv) If $K_{T}$ is a cone, then $\Xi_{t}^{\xi} \subset \Xi_{t}^{0}$ for any $\xi \in \mathcal{L}^{0}\left(K_{T}, \mathcal{F}_{T}\right)$.

Proof. (i) The infinite decomposability follows by induction and using the infinite $\mathcal{F}_{t}$-decomposability and the conditional convexity of acceptance sets. Similar arguments apply for $\hat{\Xi}_{t}^{\xi}$.

(ii) The existence of $X_{t}^{\xi}$ is trivial for $t=T$. Suppose that it holds at time $t$. The result for $t-1$ follows from the induction assumption and (5.4) by Lemma 2.8 .

(iii) follows from the fact that $\left(\gamma_{T}+\mathrm{A}_{t, T}\right) \cap\left(\xi+K_{T}\right) \neq \varnothing$ if and only if $\gamma_{T} \in\left(-\mathrm{A}_{t, T}+\xi\right)$. 
(iv) follows from (iii) by choosing $k_{T}=\xi+\tilde{k}_{T} \in \mathcal{L}^{0}\left(K_{T}, \mathcal{F}_{T}\right)$ for $\tilde{k}_{T} \in K_{T}$ a.s.

Example 5.3. If $K_{t}=\mathbb{R}_{+}^{d}$ a.s. for all $t$ (which is always the case if $d=1$ ), then an acceptable portfolio process satisfies $V_{t-1}-V_{t} \in \mathcal{C}_{t-1, t}$ for all $t=1, \ldots, T$. Then

$$
\Xi_{t}^{\xi}=\left(\xi+\sum_{s=t}^{T-1} \mathcal{C}_{s, s+1}\right) \cap \mathcal{L}^{0}\left(\mathbb{R}^{d}, \mathcal{F}_{t}\right) .
$$

If $\xi=0$ a.s., since $\mathcal{C}_{t-1, t} \cap \mathcal{L}^{0}\left(\mathbb{R}^{d}, \mathcal{F}_{t-1}\right)=\mathcal{L}^{0}\left(\mathbb{R}_{+}^{d}, \mathcal{F}_{t-1}\right)$ for all $t \geqslant 1$, the induction argument yields that $\Xi_{t}^{0}=\mathcal{L}^{0}\left(\mathbb{R}_{+}^{d}, \mathcal{F}_{t}\right)$. In this setting, $\mathrm{R}_{t, s}^{0}(\Xi)$ is called a regulator risk measure in [13]; it only takes into account the acceptability requirement and disregards any trading opportunities between the components.

\section{Risk ARBITRAGE}

Example 1.1 shows that in some cases it is possible to release infinite capital from a zero position without compromising the acceptability criterion. In order to introduce the no risk arbitrage properties, consider zero payoff $\xi=0$ and so work with families $\Xi_{t}^{0}$. By (5.5),

$$
\Xi_{t}^{0}=\left(-\mathrm{A}_{t, T}\right) \cap \mathcal{L}^{0}\left(\mathbb{R}^{d}, \mathcal{F}_{t}\right) .
$$

Definition 6.1. The multiperiod model satisfies

(SNR) (strict no risk arbitrage) if $\hat{\Xi}_{t}^{0} \cap \mathcal{L}^{0}\left(-K_{t}, \mathcal{F}_{t}\right) \subset \mathcal{L}^{0}\left(K_{t}^{0}, \mathcal{F}_{t}\right)$ for all $t=0, \ldots, T$;

(NRA) (no risk arbitrage) if $\Xi_{t}^{0} \cap \mathcal{L}^{0}\left(\mathbb{R}_{-}^{d}, \mathcal{F}_{t}\right)=\{0\}$ for all $t=0, \ldots, T$;

(NARA) (no asymptotic risk arbitrage) if $\left(\mathrm{cl}_{0} \Xi_{t}^{0}\right) \cap \mathcal{L}^{0}\left(\mathbb{R}_{-}^{d}, \mathcal{F}_{t}\right)=\{0\}$ for all $t=0, \ldots, T$;

(NRA2) (no risk arbitrage opportunity of the second kind) if $\left(\eta_{t}+\mathrm{A}_{t, T}\right) \cap$ $\mathcal{L}^{0}\left(K_{T}, \mathcal{F}_{T}\right) \neq \varnothing$ for any $t=0, \ldots, T$ and $\eta_{t} \in \mathcal{L}^{0}\left(\mathbb{R}_{+}^{d}, \mathcal{F}_{t}\right)$ yields $\eta_{t} \in \mathcal{L}^{0}\left(K_{t}, \mathcal{F}_{t}\right)+\mathcal{C}_{t-1, t}$

(SNAR) (strong no risk arbitrage) if $\sum_{t=0}^{T}\left(k_{t}+\eta_{t}\right)=0$ for $k_{t} \in \mathcal{L}^{0}\left(K_{t}, \mathcal{F}_{t}\right)$ and $\eta_{t} \in \mathcal{C}_{t-1, t}$ implies that $k_{t} \in \mathcal{L}^{0}\left(K_{t}^{0}, \mathcal{F}_{t}\right)$ and $\eta_{t}=0$ a.s. for all $t$.

It is obvious that (NARA) is stronger than (NRA). By (6.1), (NRA) condition is equivalent to $A_{t, T} \cap \mathcal{L}^{0}\left(\mathbb{R}_{+}^{d}, \mathcal{F}_{t}\right)=\{0\}$ for all $t$. Conditions (SNR) and (NRA) are violated in Example 1.1. If $K_{t}=\mathbb{R}_{+}^{d}$ a.s. for all $t$, then $\Xi_{t}^{0}=\mathcal{L}^{0}\left(\mathbb{R}_{+}^{d}, \mathcal{F}_{t}\right)$, see Example 5.3 , so that all no arbitrage conditions are satisfied. 
Lemma 6.2. (SNR) implies that

$$
\mathrm{R}_{t, t+1}\left(\Xi_{t+1}^{0}\right) \cap \mathcal{L}^{0}\left(-K_{t}, \mathcal{F}_{t}\right) \subset \mathcal{L}^{0}\left(K_{t}^{0}, \mathcal{F}_{t}\right)
$$

for all $t=0, \ldots, T-1$. The reverse implication holds if the solvency sets are strictly proper.

Proof. Denote $M=\mathrm{R}_{t, t+1}\left(\Xi_{t+1}^{0}\right), A=\mathcal{L}^{0}\left(K_{t}, \mathcal{F}_{t}\right)$, and $B=\mathcal{L}^{0}\left(K_{t}^{0}, \mathcal{F}_{t}\right)$. It is immediate to check that $M \cap(-A) \subset B$ if $(M+A) \cap(-A) \subset B$ and only if in case $A \cap(-A)=\{0\}$. For the reverse implication, if $x \in(M+A) \cap(-A)$, then $x=m+a_{1}=-a_{2}$, where $m \in M$ and $a_{1}, a_{2} \in A$. Therefore, $m / 2 \in M \cap(-A) \subset B$. Then $x / 2 \in A \cap(-A)$, so that $x \in B=A \cap(-A)=\{0\}$ if $K$ is strictly proper.

Lemma 6.3. Assume that the acceptance sets are strictly proper, that is $\mathcal{C}_{t, s} \cap\left(-\mathcal{C}_{t, s}\right)$ consists of all random vectors that equal 0 almost surely.

(i) If $K_{t}^{0}=\tilde{K}_{t}$ for all $t$, (SNAR) implies

$$
\begin{array}{ll}
\mathrm{A}_{0, t} \cap\left(\mathcal{L}^{0}\left(K_{t}, \mathcal{F}_{t}\right)+\mathcal{C}_{t-1, t}\right) \subset \mathcal{L}^{0}\left(K_{t}^{0}, \mathcal{F}_{t}\right), & t=0, \ldots, T, \\
\mathrm{~A}_{t, T} \cap\left(\mathcal{L}^{0}\left(K_{t}, \mathcal{F}_{t}\right)+\mathcal{C}_{t-1, t}\right) \subset \mathcal{L}^{0}\left(K_{t}^{0}, \mathcal{F}_{t}\right), & t=0, \ldots, T .
\end{array}
$$

(ii) If the solvency sets are strictly proper and

$$
\mathcal{L}^{0}\left(-K_{t}, \mathcal{F}_{t}\right) \cap \mathcal{C}_{t-1, t}=\{0\}, \quad t=0, \ldots, T,
$$

then each of the conditions (6.2), (6.3) implies (SNAR).

Proof. (i) Motivated by [17, Lemma 3.2.7], assume that

$$
-k_{0}-\cdots-k_{t}-\eta_{0}-\cdots-\eta_{t}=g_{t}+\zeta_{t} \in\left(\mathcal{L}^{0}\left(K_{t}, \mathcal{F}_{t}\right)+\mathcal{C}_{t-1, t}\right)
$$

with $k_{s} \in \mathcal{L}^{0}\left(K_{s}, \mathcal{F}_{s}\right), \eta_{s} \in \mathcal{C}_{s-1, s}, s=0, \ldots, t, g_{t} \in \mathcal{L}^{0}\left(K_{t}, \mathcal{F}_{t}\right)$, and $\zeta_{t} \in \mathcal{C}_{t-1, t}$. Since $\left(\eta_{t}+\zeta_{t}\right) / 2 \in \mathcal{C}_{t-1, t}$ by convexity and

$$
-k_{0} / 2-\cdots-k_{t-1} / 2-\left(k_{t}+g_{t}\right) / 2-\eta_{0} / 2-\cdots-\left(\eta_{t}+\zeta_{t}\right) / 2=0,
$$

we have $\left(k_{t}+g_{t}\right) / 2 \in K_{t}^{0}$ and $\left(\eta_{t}+\zeta_{t}\right) / 2=0$ by (SNAR). The strict properness of the acceptance sets yields that $\eta_{t}=\zeta_{t}=0$. Furthermore, $\frac{1}{2} g_{t} \in-\frac{1}{2} k_{t}+\frac{1}{2} K_{t}^{0} \subset-K_{t}$, so that $g_{t} \in K_{t}^{0}$, i.e. (6.2) holds.

Property (6.3) is similarly derived from (SNAR).

(ii) In order to show that (6.2) implies (SNAR), proceed by induction as in $\left[17\right.$, Lemma 3.2.13]. Let $-k_{0}-\cdots-k_{T}-\eta_{0}-\cdots-\eta_{T}=0$. Then

$$
k_{T}+\eta_{T}=\sum_{s=0}^{T-1}\left(-k_{s}-\eta_{s}\right) \in \mathrm{A}_{0, T-1} \subset \mathrm{A}_{0, T} .
$$

By $(6.2), k_{T}+\eta_{T} \in \mathcal{L}^{0}\left(K_{T}^{0}, \mathcal{F}_{T}\right)$. Since $k_{T}+\eta_{T}$ is $\mathcal{F}_{T-1}$-measurable and the solvency sets are strictly proper, $k_{T}+\eta_{T} \in \mathcal{L}^{0}\left(K_{T-1}^{0}, \mathcal{F}_{T-1}\right)$. Therefore, $k_{T}+\eta_{T}$ can be merged with $k_{T-1}$ and then the induction proceeds with $T-1$ instead of $T$. 
To show that (6.3) implies (SNAR), proceed by induction starting from time zero. Since

$$
k_{0}+\eta_{0}=\sum_{s=1}^{T}\left(-k_{s}-\eta_{s}\right) \in \mathrm{A}_{1, T} \subset \mathrm{A}_{0, T},
$$

(6.3) yields $k_{0}+\eta_{0}=0$ and (6.4) implies $k_{0}=\eta_{0}=0$.

Condition (6.4) can be viewed as a consistency requirement between the acceptance sets and solvency sets, namely, that $-K_{t}$ does not contain any acceptable non-trivial selection.

Theorem 6.4. If the solvency sets are proper, then (SNR) implies (NARA) and the closedness of $\hat{\Xi}_{t}^{0}$ in $\mathcal{L}^{0}$ for all $t=0, \ldots, T$.

Proof. Denote $M=\mathrm{R}_{t, t+1}\left(\Xi_{t+1}^{0}\right)$. Recall that $\mathrm{cl}_{0} \hat{\Xi}_{t}^{0}=\mathrm{cl}_{0} \Xi_{t}^{0}$. Assume that $k_{t}^{n}+\gamma_{t}^{n} \rightarrow \zeta_{t} \in \mathcal{L}^{0}\left(\mathbb{R}_{-}^{d}, \mathcal{F}_{t}\right)$ a.s. for $k_{t}^{n} \in \mathcal{L}^{0}\left(K_{t}, \mathcal{F}_{t}\right)$ and $\gamma_{t}^{n} \in M$ such that $k_{t}^{n}+\gamma_{t}^{n} \in \hat{\Xi}_{t}^{0}, n \geqslant 1$. Since $M$ is $\mathcal{L}^{0}$-closed and convex, we may assume by $\left[17\right.$, Lemma 2.1.2] that $k_{t}^{n} \rightarrow k_{t} \in \mathcal{L}^{0}\left(K_{t}, \mathcal{F}_{t}\right)$ on the set $A=\left\{\liminf _{n}\left\|k_{t}^{n}\right\|<\infty\right\}$. Hence $\gamma_{t}^{n} \rightarrow \gamma_{t} \in M$, so that

$$
\gamma_{t}=\zeta_{t}-k_{t} \in M \cap \mathcal{L}^{0}\left(-K_{t}, \mathcal{F}_{t}\right) \subset \mathcal{L}^{0}\left(K_{t}^{0}, \mathcal{F}_{t}\right) .
$$

Thus, $\gamma_{t} \in K_{t}^{0}$ and $\zeta_{t} / 2=\gamma_{t} / 2+k_{t} / 2 \in K_{t}$. Hence, $\zeta_{t} / 2 \in \mathbb{R}_{-}^{d} \cap K_{t}=\{0\}$ and $\zeta_{t}=0$.

If $\mathrm{P}(\Omega \backslash A)>0$, assume that $k_{t}^{n}=\gamma_{t}^{n}=\zeta_{t}=0$ on $A$ by $\mathcal{F}_{t^{-}}$ decomposability, and use the standard normalisation procedure, i.e. divide $k_{t}^{n}, \gamma_{t}^{n}, \zeta_{t}$ by $\left(1+\left\|k_{t}^{n}\right\|\right)$. Arguing as previously, we obtain $k_{t} \in \mathcal{L}^{0}\left(K_{t}, \mathcal{F}_{t}\right)$ such that $\left\|k_{t}\right\|=1$ on $\Omega \backslash A$. Since $0 \in M$, we have $\gamma_{t} \in M$ by conditional convexity. Moreover, $k_{t}+\gamma_{t}=0$ since $\zeta_{t} /\left(1+\left\|k_{t}^{n}\right\|\right) \rightarrow 0$. Then $\gamma_{t} \neq 0$ belongs to $M \cap \mathcal{L}^{0}\left(-K_{t}, \mathcal{F}_{t}\right)=\{0\}$, which is a contradiction in view of Lemma 6.2.

This argument also yields the closedness of $\hat{\Xi}_{t}^{0}=\mathcal{L}^{0}\left(K_{t}, \mathcal{F}_{t}\right)+M$.

The first part of the following result shows that (NRA) is similar to the weak no arbitrage property $\mathrm{NA}^{\mathrm{w}}$ of Kabanov's model, see [17, Sec. 3.2.1].

Proposition 6.5. Suppose that $\mathbb{R}_{+}^{d} \backslash\{0\} \subset \operatorname{int} K_{t}$ a.s. for all $t$. Then (NRA) and each of the following conditions are equivalent.

(i) $\mathrm{R}_{t, t+1}^{0}\left(\Xi_{t+1}^{0}\right) \cap \mathcal{L}^{0}\left(-K_{t}, \mathcal{F}_{t}\right) \subset \mathcal{L}^{0}\left(-\partial K_{t}, \mathcal{F}_{t}\right)$ for all $t$.

(ii) $\hat{\Xi}_{t}^{0} \cap \mathcal{L}^{0}\left(\mathbb{R}_{-}^{d}, \mathcal{F}_{t}\right)=\{0\}$ for all $t$.

Proof. (i) Consider $x_{t}=\gamma_{t}+k_{t}$ for $\gamma_{t} \in M=\mathrm{R}_{t, t+1}^{0}\left(\Xi_{t+1}^{0}\right)$ and $k_{t} \in$ $\mathcal{L}^{0}\left(K_{t}, \mathcal{F}_{t}\right)$. Assume that $x_{t}$ is non-trivial and $x_{t} \in \mathcal{L}^{0}\left(\mathbb{R}_{-}^{d}, \mathcal{F}_{t}\right)$. Hence 
$\gamma_{t} / 2=x_{t} / 2-k_{t} / 2 \in \mathcal{L}^{0}\left(-K_{t}, \mathcal{F}_{t}\right)$ and $\gamma_{t} / 2 \in-\operatorname{int} K_{t}$ on $\left\{x_{t} \neq 0\right\}$, since int $K_{t}$ contains $\mathbb{R}_{+}^{d} \backslash\{0\}$, contrary to the assumption.

Consider any $x_{t} \in M \cap \mathcal{L}^{0}\left(-K_{t}, \mathcal{F}_{t}\right)$ such that $x_{t}=-k_{t}$ for $k_{t} \in$ $\mathcal{L}^{0}\left(K_{t}, \mathcal{F}_{t}\right)$ such that $\mathrm{P}\left(k_{t} \in \operatorname{int} K_{t}\right)>0$. By a measurable selection argument, $k_{t}+\gamma_{t} \in \mathcal{L}^{0}\left(K_{t}, \mathcal{F}_{t}\right)$ for some $\gamma_{t} \in \mathcal{L}^{0}\left(\mathbb{R}_{-}^{d}, \mathcal{F}_{t}\right) \backslash\{0\}$. Thus,

$$
x_{t}+k_{t}+\gamma_{t}=\gamma_{t} \in\left(M+\mathcal{L}^{0}\left(K_{t}, \mathcal{F}_{t}\right)\right) \cap \mathcal{L}^{0}\left(\mathbb{R}_{-}^{d}, \mathcal{F}_{t}\right),
$$

contrary to (NRA).

(ii) It suffices to show that (NRA) implies (ii). Assume that $k_{t} \in$ $\mathcal{L}^{0}\left(K_{t}, \mathcal{F}_{t}\right)$ and $\gamma_{t} \in \mathrm{R}_{t, t+1}\left(\Xi_{t+1}^{0}\right)$ are such that $k_{t}+\gamma_{t} \in \mathbb{R}_{-}^{d}$ a.s. and $k_{t}+\gamma_{t} \neq 0$ with a positive probability. Since $k_{t} / 2+\mathbb{R}_{+}^{d} \subset\left(\left\{k_{t} / 2\right\} \cup\right.$ $\left.\operatorname{int} K_{t}\right)$ a.s., the set (int $\left.K_{t}+\mathrm{R}_{t, t+1}\left(\Xi_{t+1}^{0}\right)\right)$ has a non-trivial intersection with $\mathbb{R}_{-}^{d}$ with a positive probability. By Proposition 2.5 with $X=$ $\operatorname{int} K_{t}$ and $\Xi=\mathrm{R}_{t, t+1}^{0}\left(\Xi_{t+1}^{0}\right)$, the set (int $K_{t}+\mathrm{R}_{t, t+1}^{0}\left(\Xi_{t+1}^{0}\right)$ ) has a nontrivial intersection with $\mathbb{R}_{-}^{d}$ with a positive probability, contrary to (NRA).

Theorem 6.6. Assume that the acceptance sets are continuous from below. Let $p \in[1, \infty]$.

(i) (NARA) is equivalent to

$$
\overline{\mathrm{A}}_{t, T}^{p} \cap \mathcal{L}^{0}\left(\mathbb{R}_{+}^{d}, \mathcal{F}_{t}\right)=\{0\}, \quad t=0, \ldots, T-1 .
$$

(ii) If the solvency sets are proper, then (SNR) is equivalent to

$$
\overline{\mathrm{A}}_{t, T}^{p} \cap \mathcal{L}^{0}\left(K_{t}, \mathcal{F}_{t}\right)=\{0\}, \quad t=0, \ldots, T-1 .
$$

Proof. (i) Given (6.1), it suffices to show that (NARA) is equivalent to

$$
\operatorname{cl}_{p}\left(\Xi_{t}^{0} \cap \mathcal{L}^{p}\left(\mathbb{R}^{d}, \mathcal{F}_{t}\right)\right) \cap \mathcal{L}^{0}\left(\mathbb{R}_{-}^{d}, \mathcal{F}_{t}\right)=\{0\}, \quad t=0, \ldots, T-1 .
$$

Assume (6.7) and consider $x_{t} \in\left(\mathrm{cl}_{0} \Xi_{t}^{0}\right) \cap \mathcal{L}^{0}\left(\mathbb{R}_{-}^{d}, \mathcal{F}_{t}\right)$. Then $x_{t}^{n} \rightarrow x_{t}$ a.s. for $x_{t}^{n} \in \Xi_{t}^{0}, n \geqslant 1$. Hence,

$$
x_{t}^{n} \mathbf{1}_{\left\|x_{t}^{n}\right\| \leqslant m+1} \mathbf{1}_{\left\|x_{t}\right\| \leqslant m} \rightarrow x_{t} \mathbf{1}_{\left\|x_{t}\right\| \leqslant m} \quad \text { as } n \rightarrow \infty
$$

for all $m \geqslant 1$, where $x_{t}^{n} \mathbf{1}_{\left\|x_{t}^{n}\right\| \leqslant m+1} \mathbf{1}_{\left\|x_{t}\right\| \leqslant m} \in \Xi_{t}^{0}$ by decomposability and since $0 \in \Xi_{t}^{0}$. The dominated convergence theorem yields that $x_{t} \mathbf{1}_{\left\|x_{t}\right\| \leqslant m}$ belongs to $\operatorname{cl}_{p}\left(\Xi_{t}^{0} \cap \mathcal{L}^{p}\right) \cap \mathcal{L}^{0}\left(\mathbb{R}_{-}^{d}, \mathcal{F}_{t}\right)=\{0\}$. Letting $m \rightarrow \infty$ yields $x_{t}=0$, i.e. (NARA) holds.

Reciprocally, assume (NARA). Consider a sequence $\left(V_{t, T}^{n}\right)_{n \geqslant 1}$ from $\mathrm{A}_{t, T}^{p}$ which converges in $\mathcal{L}^{p}$ to $z_{t}^{+} \in \mathcal{L}^{0}\left(\mathbb{R}_{+}^{d}, \mathcal{F}_{t}\right)$. Passing to a subsequence, assume that $V_{t, T}^{n} \rightarrow z_{t}^{+}$in $\mathcal{L}_{\mathcal{F}_{t}}^{p}$ and a.s. Then

$$
\gamma_{t}^{n}=\left(V_{t, T}^{n}-z_{t}^{+}\right) \mathbf{1}_{\left\|V_{t, T}^{n}\right\| \leqslant\left\|z_{t}^{+}\right\|+1} \rightarrow 0 \quad \text { in } \quad \mathcal{L}_{\mathcal{F}_{t}}^{p} \quad \text { and a.s. }
$$


The same fact holds for the $\mathcal{F}_{t}$-bounded increasing sequence $\tilde{\gamma}_{t}^{n}=$ $\inf _{m \geqslant n} \gamma_{t}^{n}$, where the infimum is taken componentwise. Observe that $\tilde{\gamma}_{t}^{n}=\gamma_{t}^{n}-\varepsilon_{T}^{n}$ with $\varepsilon_{T}^{n} \in \mathcal{L}^{0}\left(\mathbb{R}_{+}^{d}, \mathcal{F}_{T}\right), n \geqslant 1$. Therefore, if we define

$$
\tilde{V}_{t, T}^{n}=V_{t, T}^{n} \mathbf{1}_{\left\|V_{t, T}^{n}\right\| \leqslant\left\|z_{t}^{+}\right\|+1}-\varepsilon_{T}^{n},
$$

then

$$
\tilde{\gamma}_{t}^{n}=\left(\tilde{V}_{t, T}^{n}-z_{t}^{+}\right) \mathbf{1}_{\left\|V_{t, T}^{n}\right\| \leqslant\left\|z_{t}^{+}\right\|+1}
$$

is the terminal value of a portfolio process. Note that $\tilde{\gamma}_{t}^{1} \geqslant-c_{t} \mathbf{1}$, where $c_{t}=\left\|z_{t}^{+}\right\|+1$. By the continuity from above, since $0 \in \mathcal{C}_{T-1, T}$, there exists a decreasing sequence $\zeta_{T-1}^{n} \in \mathcal{L}^{0}\left(\mathbb{R}^{d}, \mathcal{F}_{T-1}\right)$ which converges a.s. to 0 and such that $\eta_{T}^{n}=\tilde{\gamma}^{n}+\zeta_{T-1}^{n} \in \mathcal{C}_{T-1, T}$ for all $n$. In view of Definition 4.5, assume that $\zeta_{T-1}^{n} \leqslant c_{t} \mathbf{1}$, for all $n$, so that $\zeta_{T-1}^{n} \rightarrow 0$ in $\mathcal{L}_{\mathcal{F}_{T-2}}^{p}$ if $T-2 \geqslant t$. Since $0 \in \mathcal{C}_{T-2, T-1}$ and $-\zeta_{T-1}^{n} \uparrow 0$ in $\mathcal{L}_{\mathcal{F}_{T-2}}^{p}$, the continuity from above property yields the existence of a decreasing sequence $\zeta_{T-2}^{n} \in \mathcal{L}^{0}\left(\mathbb{R}^{d}, \mathcal{F}_{T-2}\right)$ which converges a.s. to 0 and such that $\eta_{T-1}^{n}=-\zeta_{T-1}^{n}+\zeta_{T-2}^{n} \in \mathcal{C}_{T-2, T-1}$ for all $n$. Moreover, $\zeta_{T-2}^{n} \leqslant c_{t} \mathbf{1}$, so that $\zeta_{T-1}^{n} \rightarrow 0$ in $\mathcal{L}_{\mathcal{F}_{T-3}}^{p}$ if $T-3 \geqslant t$. Therefore, we may reiterate the construction and find $\zeta_{T-3}^{n}, \ldots, \zeta_{t}^{n}$ such that $\zeta_{t}^{n} \rightarrow 0$ a.s. Then $\eta_{u+1}^{n}=-\zeta_{u+1}^{n}+\zeta_{u}^{n} \in \mathcal{C}_{u, u+1}$ if $t \leqslant u \leqslant T-2$. Since $\tilde{\gamma}_{t}^{n}+\zeta_{t}^{n}=\sum_{u=t}^{T-1} \eta_{u+1}^{n}$,

$$
\tilde{\gamma}_{t}^{n}+\zeta_{t}^{n} \in \mathcal{C}_{t, t+1}+\cdots+\mathcal{C}_{T-1, T}
$$

Notice that

$$
\frac{1}{2}\left(-z_{t}^{+} \mathbf{1}_{\left\|V_{t, T}^{n}\right\| \leqslant\left\|z_{t}^{+}\right\|+1}+\zeta_{t}^{n}\right)=-\frac{1}{2} \tilde{V}_{t, T}^{n}+\frac{1}{2}\left(\tilde{\gamma}_{t}^{n}+\zeta_{t}^{n}\right) .
$$

By convexity,

$$
\frac{1}{2}\left(-z_{t}^{+} \mathbf{1}_{\left\|V_{t, T}^{n}\right\| \leqslant\left\|z_{t}^{+}\right\|+1}+\zeta_{t}^{n}\right) \in \Xi_{t}^{0} .
$$

Letting $n \rightarrow \infty$ yields that $-\frac{1}{2} z_{t}^{+} \in\left(\mathrm{cl}_{0} \Xi_{t}^{0}\right) \cap \mathcal{L}^{0}\left(-\mathbb{R}_{+}^{d}, \mathcal{F}_{t}\right)$, so that $z_{t}^{+}=0$ by (NARA). Thus, (6.5) and (6.5) hold.

(ii) Recall that $\hat{\Xi}_{t}^{0}=\operatorname{cl}_{0}\left(\hat{\Xi}_{t}^{0}\right)=\operatorname{cl}_{0}\left(\Xi_{t}^{0}\right)$ by Theorem 6.4. Following the arguments from (i), we obtain that (SNR) is equivalent to

$$
\operatorname{cl}_{p}\left(\Xi_{t}^{0} \cap \mathcal{L}^{p}\left(\mathbb{R}^{d}, \mathcal{F}_{t}\right)\right) \cap \mathcal{L}^{p}\left(-K_{t}, \mathcal{F}_{t}\right)=\{0\}, \quad t=0, \ldots, T-1 .
$$

In view of (6.1), $\operatorname{cl}_{p}\left(\Xi_{t}^{0} \cap \mathcal{L}^{p}\right) \subset-\overline{\mathrm{A}}_{t, T}^{p}$. Therefore, (6.6) implies (6.8) and (SNR) holds.

Reciprocally, assume (SNR). Consider a sequence $\left(V_{t, T}^{n}\right)_{n \geqslant 1}$ from $\mathrm{A}_{t, T}^{p}$ which converges in $\mathcal{L}^{p}$ to $k_{t} \in \mathcal{L}^{0}\left(K_{t}, \mathcal{F}_{t}\right)$ and follow the proof of (i) with $k_{t}$ instead of $z_{t}^{+}$. 
Theorem 6.7. If the solvency sets are proper and the acceptance sets are continuous from below, then (SNR) yields that $\hat{\Xi}_{t}^{\xi}$ is closed in $\mathcal{L}^{0}$ for all $t$ and any $\xi \in \mathcal{L}^{p}\left(\mathbb{R}^{d}, \mathcal{F}_{T}\right)$, so that $\hat{\Xi}_{t}^{\xi}=\mathcal{L}^{0}\left(X_{t}^{\xi}, \mathcal{F}_{t}\right)$ for random closed sets $X_{t}^{\xi}, t=0, \ldots, T$.

Proof. Assume that $k_{t}^{n}+\gamma_{t}^{n} \rightarrow \zeta_{t} \in \mathcal{L}^{0}\left(\mathbb{R}^{d}, \mathcal{F}_{t}\right)$ a.s. for $k_{t}^{n} \in \mathcal{L}^{0}\left(K_{t}, \mathcal{F}_{t}\right)$ and $\gamma_{t}^{n} \in M=\mathrm{R}_{t, t+1}\left(\Xi_{t+1}^{\xi}\right)$ such that $k_{t}^{n}+\gamma_{t}^{n} \in \hat{\Xi}_{t}^{0}$. Since $M$ is $\mathcal{L}^{0}-$ closed and convex, we may assume by [17, Lemma 2.1.2] that $k_{t}^{n} \rightarrow k_{t} \in$ $\mathcal{L}^{0}\left(K_{t}, \mathcal{F}_{t}\right)$ on the set $A=\left\{\liminf _{n}\left\|k_{t}^{n}\right\|<\infty\right\}$. Hence, $\gamma_{t}^{n} \rightarrow \gamma_{t} \in M$, so that $\zeta_{t}=k_{t}+\gamma_{t} \in \hat{\Xi}_{t}^{\xi}$.

If $\mathrm{P}(\Omega \backslash A)>0$, assume that $k_{t}^{n}=\gamma_{t}^{n}=\zeta_{t}=0$ on $A$ by $\mathcal{F}_{t^{-}}$ decomposability, and use the normalisation procedure, i.e. obtain $\tilde{k}_{t}^{n}$ and $\tilde{\gamma}_{t}^{n}$ by scaling $k_{t}^{n}$ and $\gamma_{t}^{n}$ with $c_{t}^{n}=\left(1+\left\|k_{t}^{n}\right\|\right)^{-1}$. We may assume that $\left\|\tilde{\gamma}_{t}^{n}\right\| \leqslant 2$, since $c_{t}^{n} \zeta_{t} \rightarrow 0$, so that $\tilde{k}_{t}^{n}+\tilde{\gamma}_{t}^{n} \rightarrow 0$. Arguing as previously, $\tilde{k}_{t}^{n} \rightarrow \tilde{k}_{t} \in \mathcal{L}^{0}\left(K_{t}, \mathcal{F}_{t}\right)$ in $\mathcal{L}^{p}$, and $\left\|\tilde{k}_{t}\right\|=1$ on $\Omega \backslash A$. Therefore, $\tilde{\gamma}_{t}^{n} \rightarrow \tilde{\gamma}_{t}=-\tilde{k}_{t}$ in $\mathcal{L}^{p}$, so that $\tilde{k}_{t}+\tilde{\gamma}_{t}=0$. Notice that $M=\operatorname{cl}_{0}\left(\left(-\mathrm{A}_{t+1, T}+\xi\right) \cap \mathcal{L}^{0}\left(\mathbb{R}^{d}, \mathcal{F}_{t}\right)\right)$. By convexity,

$$
\tilde{\gamma}_{t}^{n} \in \operatorname{cl}_{0}\left(-\mathrm{A}_{t+1, T}+c_{t}^{n} \xi\right) \cap \mathcal{L}^{0}\left(\mathbb{R}^{d}, \mathcal{F}_{t}\right) .
$$

Since $\left\|\tilde{\gamma}_{t}^{n}\right\| \leqslant 2$, assume without loss of generality that $\tilde{\gamma}_{t}^{n} \in$ $\mathrm{cl}_{p}\left(-\mathrm{A}_{t+1, T}+c_{t}^{n} \xi\right)$. To see this, it suffices to multiply the sequence $\left(\bar{\gamma}_{t}^{m}\right)_{m \geqslant 1}$ approximating $\tilde{\gamma}_{t}^{n}$ by $\mathbf{1}_{\left\|\bar{\gamma}_{t}^{m}\right\| \leqslant 3}$. Letting $n \rightarrow \infty$, (6.6) yields that

$$
-\tilde{\gamma}_{t} \in \overline{\mathrm{A}}_{t, T}^{p} \cap \mathcal{L}^{0}\left(K_{t}, \mathcal{F}_{t}\right)=\{0\} .
$$

Thus, $\gamma_{t}=0$, so that $\mathrm{P}(\Omega \backslash A)=0$ and the conclusion follows.

Lemma 6.8. (NRA2) is equivalent to

$$
\Xi_{t}^{\xi} \subset \mathcal{L}^{0}\left(K_{t}, \mathcal{F}_{t}\right)+\mathcal{C}_{t-1, t}, \quad t=0, \ldots, T,
$$

for any $\xi \in \mathcal{L}^{0}\left(K_{T}, \mathcal{F}_{T}\right)$. If (6.4) holds, then (NRA2) implies (NARA).

Proof. Note that $\left(\eta_{t}+\mathrm{A}_{t, T}\right)$ intersects $\mathcal{L}^{0}\left(K_{T}, \mathcal{F}_{T}\right)$ if and only if

$$
\eta_{t} \in\left(-\mathrm{A}_{t, T}+\mathcal{L}^{0}\left(K_{T}, \mathcal{F}_{T}\right)\right) \cap \mathcal{L}^{0}\left(\mathbb{R}^{d}, \mathcal{F}_{t}\right),
$$

that is $\eta_{t} \in \Xi_{t}^{\xi}$ for some $\xi=k_{T} \in \mathcal{L}^{0}\left(K_{T}, \mathcal{F}_{T}\right)$.

Definition 6.9. Assume that the solvency sets are conical. For $t \leqslant T$, an adapted process $Z=\left(Z_{s}\right)_{s=t, \ldots, T}$ is a $q$-integrable $t$-weakly consistent price system if it is a $\mathrm{Q}$-martingale for $\mathrm{Q} \sim \mathrm{P}$ such that $Z_{s}$ is a $q$ integrable under $\mathrm{Q}, \mathcal{F}_{s}$-measurable selection of $K_{s}^{*}$ for every $s \geqslant t$ and $\mathrm{E}_{\mathrm{Q}}\left(Z_{T} \mid \mathcal{F}_{t}\right) \in K_{t}^{*} \backslash\{0\}$. We denote by $\mathcal{M}_{t, T}^{q, w}(\mathrm{Q})$ the set all $q$-integrable $t$-weakly consistent price systems under $Q$, where $q \in[1, \infty]$. 
Theorem 6.10. Assume the coherent conical setting and that the solvency sets are continuous from below. Let $q$ be the conjugate of $p$ that stems from the definition of the acceptance sets.

(i) (NARA) is equivalent to the fact that, for each $t$, there exists $Z \in \mathcal{M}_{t, T}^{q, w}(\mathrm{P})$ such that

$$
\mathrm{E}\left\langle Z_{u}, \eta_{u}\right\rangle \geqslant 0 \text { for all } \eta_{u} \in \mathcal{C}_{u-1, u}, u=t+1, \ldots, T \text {. }
$$

(ii) If int $K_{t}^{*} \neq \varnothing$ a.s. for all $t$, then (SNR) is equivalent to the fact that, for each $t$, there exists $Z \in \mathcal{M}_{t, T}^{q, w}(\mathrm{P})$ such that (6.10) holds and $Z_{t} \in \mathcal{L}^{0}\left(\operatorname{int} K_{t}^{*}, \mathcal{F}_{t}\right)$.

Proof. (i) The existence of $Z \in \mathcal{M}_{t, T}^{q, w}(\mathrm{P})$ such that $\mathrm{E}\left\langle Z_{t}, \eta\right\rangle \geqslant 0$ for all $\eta \in \mathcal{C}_{t, t+1}+\cdots+\mathcal{C}_{T-1, T}$ is a direct consequence of the Hahn-Banach separation theorem and Theorem 6.6(i).

Reciprocally, for each $t$, assume the existence of $Z \in \mathcal{M}_{t, T}^{q, w}(\mathrm{P})$ and consider $x_{T} \in \mathrm{A}_{t, T}^{p}$. Then

$$
x_{T}=-k_{t}-\left(k_{t+1}+\eta_{t+1}\right)-\cdots-\left(k_{T}+\eta_{T}\right),
$$

where $\eta_{s} \in \mathcal{C}_{s-1, s}$ and $k_{s} \in \mathcal{L}^{0}\left(K_{s}, \mathcal{F}_{s}\right)$ for $s \geqslant t$. Since $\eta_{s}=\eta_{s}^{\prime}+\eta_{s}^{\prime \prime}$, where $\eta_{s}^{\prime} \in \mathcal{C}_{s-1, s} \cap \mathcal{L}_{\mathcal{F}_{s-1}}^{p}\left(\mathbb{R}^{d}, \mathcal{F}_{s}\right)$ and $\eta_{s}^{\prime \prime} \in \mathcal{L}^{0}\left(\mathbb{R}_{+}^{d}, \mathcal{F}_{s}\right)$, we may merge $\eta_{s}^{\prime \prime}$ and $k_{s}$ and suppose without loss of generality that $\eta_{s}=\eta_{s}^{\prime}$.

Using the backward induction on $t \leqslant T$, we show that $\mathrm{E}\left\langle Z_{T}, x_{T}\right\rangle \leqslant 0$. If $x_{T}=-k_{T-1}-k_{T}-\eta_{T}$, this is trivial. Since $\eta_{t+1}, k_{t} \in \mathcal{L}_{\mathcal{F}_{t}}^{p}\left(\mathbb{R}^{d}, \mathcal{F}_{t+1}\right)$, there exists a partition $\left(B_{t}^{i}\right)_{i \geqslant 1}$ from $\mathcal{F}_{t}$ such that $\eta_{t+1} \mathbf{1}_{B_{t}^{i}}, k_{t} \mathbf{1}_{B_{t}^{i}} \in$ $\mathcal{L}^{p}\left(\mathbb{R}^{d}, \mathcal{F}_{t}\right)$ for all $i \geqslant 1$. Then

$$
x_{t+1}^{i}=\left(-k_{t+1}-\cdots-k_{T}-\eta_{t+2}-\cdots-\eta_{T}\right) \mathbf{1}_{B_{t}^{i}} \in \mathrm{A}_{t+1, T}^{p}, \quad i \geqslant 1 .
$$

Moreover,

$$
\begin{aligned}
\mathrm{E}\left\langle Z_{T}, x_{T}\right\rangle & =\sum_{i=1}^{\infty} \mathrm{E}\left\langle Z_{T}, x_{t+1}^{i}\right\rangle+\sum_{i=1}^{\infty} \mathrm{E}\left\langle Z_{T},-k_{t} \mathbf{1}_{B_{t}^{i}}\right\rangle+\mathrm{E}\left\langle Z_{t},-\eta_{t+1}\right\rangle \mathbf{1}_{B_{t}^{i}}, \\
& =\sum_{i=1}^{\infty} \mathrm{E}\left\langle Z_{T}, x_{t+1}^{i}\right\rangle+\sum_{i=1}^{\infty} \mathrm{E}\left\langle Z_{t},-k_{t} \mathbf{1}_{B_{t}^{i}}\right\rangle+\mathrm{E}\left\langle Z_{t+1},-\eta_{t+1} \mathbf{1}_{B_{t}^{i}}\right\rangle \\
& \leqslant \sum_{i=1}^{\infty} \mathrm{E}\left\langle Z_{T}, x_{t+1}^{i}\right\rangle .
\end{aligned}
$$

The induction hypothesis yields that $\mathrm{E}\left\langle Z_{T}, x_{t+1}^{i}\right\rangle \leqslant 0$. Hence, $\mathrm{E}\left\langle Z_{T}, x_{T}\right\rangle \leqslant 0$. Therefore, $\mathrm{E}\left\langle Z_{T}, x_{T}\right\rangle \leqslant 0$ for all $x_{T} \in \overline{\mathrm{A}}_{t, T}^{p}$. In particular, if $x_{T}=x_{t} \in \mathcal{L}^{0}\left(\mathbb{R}_{+}^{d}, \mathcal{F}_{t}\right)$, then $\mathrm{E}\left\langle Z_{t}, x_{t}\right\rangle \leqslant 0$ and finally $\mathrm{E}\left\langle Z_{t}, x_{t}\right\rangle=0$. Since $Z_{t} \in \operatorname{int} \mathbb{R}_{+}^{d}$, we have $x_{T}=0$, i.e. (NARA) holds by Theorem 6.6(i). 
(ii) We follow the proof of (i) using the Hahn-Banach theorem and following the arguments of [19, Th. 4.1] in order to construct $Z \in$ $\mathcal{M}_{t, T}^{q, w}(\mathrm{P})$ such that $Z_{t} \in \mathcal{L}^{0}\left(\operatorname{int} K_{t}^{*}, \mathcal{F}_{t}\right)$.

\section{Conditional Core as Risk measure}

Assume that $\mathcal{C}_{t, s}=\mathcal{L}^{0}\left(\mathbb{R}_{+}^{d}, \mathcal{F}_{s}\right)$ for all $0 \leqslant t \leqslant s \leqslant T$ and $p=\infty$, so that $\mathrm{R}_{t, s}^{0}(\Xi)=\Xi \cap \mathcal{L}^{0}\left(\mathbb{R}^{d}, \mathcal{F}_{t}\right)$ for any upper set $\Xi \subset \mathcal{L}^{0}\left(\mathbb{R}^{d}, \mathcal{F}_{s}\right)$. If $X$ is an upper random closed set, then

$$
\mathrm{R}_{t, s}^{0}(X)=\mathrm{R}_{t, s}(X)=\mathbf{m}\left(X \mid \mathcal{F}_{t}\right)
$$

that is the dynamic selection risk measure is the conditional core. An acceptable portfolio process is characterised by $V_{t-1}-V_{t} \in K_{t}$ a.s. for $t=1, \ldots, T$. Then

$$
\mathrm{A}_{t, s}=\sum_{u=t}^{s} \mathcal{L}^{0}\left(-K_{u}, \mathcal{F}_{u}\right), \quad t \leqslant s,
$$

is the set of claims attainable at time $s$ starting from zero endowment at time $t$. This expression is well known in the theory of markets with transaction costs [17].

Consider the claim $\xi$ and the sets $\Xi_{t}^{\xi}$ and $\hat{\Xi}_{t}^{\xi}$ defined in Section 5 . Note that $\Xi_{t}^{\xi}$ is the set of superhedging prices that was used in [20] to define a risk measure of $\xi$.

All no risk arbitrage conditions in this section are understood with respect to the conditional core taken as the risk measure, and so with the attainable sets of claims given by (7.1). The classical no arbitrage condition $\left(\mathrm{NA}^{\mathrm{s}}\right)$ (no strict arbitrage opportunity at any time), see $[17$, Sec. 3.1.4], then becomes (6.2). (SNA) (strong no arbitrage), see Condition (iii) in [17, Sec. 3.2.2], has the same formulation as (SNAR) for the chosen acceptance sets.

Theorem 7.1. Suppose that the solvency sets $\left(K_{t}\right)_{t=0, \ldots, T}$ are strictly proper. Then (SNR), $\left(\mathrm{NA}^{\mathrm{s}}\right)$ and $(\mathrm{SNA})$ are all equivalent and are also equivalent to each of the following conditions.

(i) $\overline{\mathrm{A}}_{t, T}^{p} \cap \mathcal{L}^{0}\left(K_{t}, \mathcal{F}_{t}\right)=\{0\}$, for all $t \leqslant T-1$.

(ii) $\mathrm{A}_{t, T}$ is closed in $\mathcal{L}^{0}$ and $\mathrm{A}_{t, T} \cap \mathcal{L}^{0}\left(K_{t}, \mathcal{F}_{t}\right)=\{0\}$, for all $t \leqslant T-1$.

Proof. (SNR) is equivalent to (i) by Theorem 6.6(ii). The equivalence of $\left(\mathrm{NA}^{\mathrm{s}}\right)$ and (SNA) follows from Lemma 6.3 given that (6.4) trivially holds.

The implication (i) $\Rightarrow$ (ii) is simple to show by induction. First, $\mathrm{A}_{T, T}$ is closed. Assume that $-k_{t}^{n}-\cdots-k_{T}^{n} \rightarrow \xi$ a.s. for $k_{u}^{n} \in \mathcal{L}^{0}\left(K_{u}, \mathcal{F}_{u}\right)$, $u \geqslant t$. On the set $\left\{\liminf _{n}\left\|k_{t}^{n}\right\|=\infty\right\}$, we use the normalisation 
procedure to arrive at a contradiction with (i). Otherwise, suppose that $-k_{t}^{n} \rightarrow-k_{t} \in-K_{t}$, so that we may use the induction hypothesis to conclude.

In order to derive the closedness of $A_{t, r}$ under (SNA), it suffices to follow the proof of [17, Lemma 3.2.8]. Indeed, since $K_{t}^{0}$ is a linear space, the recession cone

$$
K_{t}^{\infty}=\bigcap_{\alpha>0} \alpha K_{t}=\left\{x \in \mathbb{R}^{d}: K_{t}+\alpha x \subset K_{t} \forall \alpha>0\right\} .
$$

satisfies $K_{t}^{0} \subset K_{t}^{\infty}$, see [23]. Therefore, $k_{t}+\alpha x \in K_{t}$ for all $k_{t} \in$ $K_{t}, x \in K_{t}^{0}$, and all $\alpha \in \mathbb{R}$. Furthermore, (SNA) trivially implies $\mathrm{A}_{t, T} \cap \mathcal{L}^{0}\left(K_{t}, \mathcal{F}_{t}\right)=\{0\}$ for all $t$.

In order to show that (ii) implies $\left(\mathrm{NA}^{\mathrm{s}}\right)$, assume

$$
-k_{0}-\cdots-k_{t}=\tilde{k}_{t} \in \mathrm{A}_{0, t} \cap \mathcal{L}^{0}\left(K_{t}, \mathcal{F}_{t}\right) .
$$

Then $k_{0} \in \mathrm{A}_{0, T} \cap \mathcal{L}^{0}\left(K_{0}, \mathcal{F}_{0}\right)$, i.e. $k_{0}=0$ by (ii). Similarly, $k_{1}=\cdots=$ $k_{t-1}=0$, so that $-k_{t}=\tilde{k}_{t}=0$, since $K_{t}$ is strictly proper. Thus, $\left(\mathrm{NA}^{\mathrm{s}}\right)$ holds.

At last, $\left(\mathrm{NA}^{\mathrm{s}}\right.$ ) yields (SNA), so that $\mathrm{A}_{t, T}$ is closed in $\mathcal{L}^{0}$. Finally, (SNA) yields (6.3) and so $\mathrm{A}_{t, T} \cap \mathcal{L}^{0}\left(K_{t}, \mathcal{F}_{t}\right)=\{0\}$, that is (i) holds.

(NA2) (no arbitrage opportunity of the second kind) from [24] and $[17$, p. 135] has the same formulation as (NRA2).

Lemma 7.2. Assume that the solvency sets are cones.

(i) (NA2) is equivalent to $\Xi_{t}^{0}=\mathcal{L}^{0}\left(K_{t}, \mathcal{F}_{t}\right)$ for all $t \leqslant T$.

(ii) (NA2) is equivalent to

$$
\mathbf{m}\left(K_{t} \mid \mathcal{F}_{t-1}\right) \subset K_{t-1}, \quad t=1, \ldots, T .
$$

(iii) If the solvency sets are strictly proper, then (NA2) implies (SNR).

Proof. (i) By Lemma 6.8 and Lemma 5.2(iv), $\Xi_{t}^{0}=\mathcal{L}^{0}\left(K_{t}, \mathcal{F}_{t}\right)$ yields (6.9), and so implies (NA2). In the other direction, (NA2) yields that $\Xi_{t}^{0} \subset \mathcal{L}^{0}\left(K_{t}, \mathcal{F}_{t}\right)+\mathcal{C}_{t-1, t}$, while (5.3) and the choice of $\mathcal{C}_{t-1, t}$ yields that $\Xi_{t}^{0} \supset \mathcal{L}^{0}\left(K_{t}, \mathcal{F}_{t}\right)$.

(ii) If $\Xi_{t}^{0} \subset \mathcal{L}^{0}\left(K_{t}, \mathcal{F}_{t}\right)$, then

$$
\Xi_{t+1}^{0} \cap \mathcal{L}^{0}\left(\mathbb{R}^{d}, \mathcal{F}_{t}\right) \subset \mathcal{L}^{0}\left(K_{t}, \mathcal{F}_{t}\right)
$$

for all $t$ by (5.3). Since $\mathcal{L}^{0}\left(K_{t+1}, \mathcal{F}_{t+1}\right) \subset \Xi_{t+1}^{0}$, we obtain (7.2).

If (7.2) holds, then

$$
\Xi_{T-1}^{0}=\mathcal{L}^{0}\left(K_{T-1}, \mathcal{F}_{T-1}\right)+\mathbf{m}\left(K_{T} \mid \mathcal{F}_{T-1}\right) \subset \mathcal{L}^{0}\left(K_{T-1}, \mathcal{F}_{T-1}\right) .
$$

Assume that $\Xi_{s}^{0} \subset \mathcal{L}^{0}\left(K_{s}, \mathcal{F}_{s}\right)$ for $s=t+1, \ldots, T$. Then

$$
\Xi_{t}^{0}=\mathcal{L}^{0}\left(K_{t}, \mathcal{F}_{t}\right)+\left(\Xi_{t+1}^{0} \cap \mathcal{L}^{0}\left(\mathbb{R}^{d}, \mathcal{F}_{t}\right)\right)
$$




$$
\subset \mathcal{L}^{0}\left(K_{t}, \mathcal{F}_{t}\right)+\mathbf{m}\left(K_{t+1} \mid \mathcal{F}_{t}\right) \subset \mathcal{L}^{0}\left(K_{t}, \mathcal{F}_{t}\right)
$$

The proof is finished by the induction argument.

(iii) Since $\Xi_{t}^{0}$ is closed in probability under (NRA2), $\Xi_{t}^{0}=\hat{\Xi}_{t}^{0}$ and (SNR) means that $\mathcal{L}^{0}\left(K_{t}, \mathcal{F}_{t}\right) \cap \mathcal{L}^{0}\left(-K_{t}, \mathcal{F}_{t}\right)=\{0\}$, which is the case if the solvency sets are strictly proper.

For conical solvency sets satisfying $\mathbf{m}\left(K_{t}^{0} \mid \mathcal{F}_{t-1}\right) \subset K_{t-1}^{0}, t \leqslant T$, in particular, for strictly proper ones, $\left(\mathrm{NA}^{\mathrm{s}}\right)$ is equivalent to the existence of a Q-martingale evolving in the relative interior of $\left(K_{t}^{*}\right)_{t=0, \ldots, T}$ for a probability measure $\mathrm{Q}$ equivalent to $\mathrm{P}$, see [17, Theorem 3.2.2]. Such a martingale is called a strictly consistent price system. If int $K_{t}^{*} \neq$ for all $t$, this result follows from Theorem 6.10(ii).

Note that $\Xi_{T}^{\xi}=\mathcal{L}^{0}\left(X_{T}^{\xi}, \mathcal{F}_{T}\right)$ with $X_{T}=\xi+K_{T}$, and $\Xi_{T-1}^{\xi}=$ $\mathcal{L}^{0}\left(X_{T-1}^{\xi}, \mathcal{F}_{T-1}\right)$ is the family of selections for a possibly non-closed random set $X_{T-1}^{\xi}=K_{T-1}+\mathbf{m}\left(X_{T}^{\xi} \mid \mathcal{F}_{T-1}\right)$. One needs additional assumptions of the no arbitrage type in order to extend this interpretation for $\Xi_{t}^{\xi}$ with $t \leqslant T-2$. Precisely the sum above should be closed, so that $\mathbf{m}\left(X_{t}^{\xi} \mid \mathcal{F}_{t-1}\right)$ exists for $t \leqslant T-1$, which makes it possible to apply Lemma 2.8 .

Theorem 7.3. Assume that the solvency sets are strictly proper and $\left(\mathrm{NA}^{\mathrm{s}}\right)$ holds. Then $\Xi_{t}^{\xi}=\mathcal{L}^{0}\left(X_{t}^{\xi}, \mathcal{F}_{t}\right)$, where $X_{t}^{\xi}$ is a $\mathcal{F}_{t}$-measurable random closed convex set, $t=0, \ldots, T$, such that $X_{T}^{\xi}=\xi+K_{T}$, and

$$
X_{t}^{\xi}=K_{t}+\mathbf{m}\left(X_{t+1}^{\xi} \mid \mathcal{F}_{t}\right), \quad t=T-1, \ldots, 0 .
$$

Proof. It suffices to confirm the statement for $t=T-1$ and then use the induction. Indeed, by Theorem $7.1,\left(\mathrm{NA}^{\mathrm{s}}\right)$ is equivalent to (SNR) so that Theorem 6.7 applies. Since $\Xi_{t}^{\xi}$ is $\mathcal{F}_{t}$-decomposable, Corollary 2.3 yields the existence of an $\mathcal{F}_{T-1}$-measurable closed set $X_{T-1}^{\xi}$ such that $\Xi_{T-1}^{\xi}=\mathcal{L}^{0}\left(X_{T-1}^{\xi}, \mathcal{F}_{T-1}\right)$. Since $X_{T}^{\xi}$ is closed,

$$
\begin{aligned}
\Xi_{T-1}^{\xi} & =\mathcal{L}^{0}\left(K_{T-1}, \mathcal{F}_{T-1}\right)+\mathcal{L}^{0}\left(\mathbf{m}\left(X_{T}^{\xi} \mid \mathcal{F}_{T-1}\right), \mathcal{F}_{T-1}\right), \\
& =\mathcal{L}^{0}\left(K_{T-1}+\mathbf{m}\left(X_{T}^{\xi} \mid \mathcal{F}_{T-1}\right), \mathcal{F}_{T-1}\right) \\
& =\mathcal{L}^{0}\left(X_{T-1}^{\xi}, \mathcal{F}_{T-1}\right),
\end{aligned}
$$

is a random set by Lemma 2.8 .

Proposition 7.4. Suppose that the solvency sets are strictly proper. Then $\left(\mathrm{NA}^{\mathrm{s}}\right)$ holds if and only if $\Xi_{t}^{0}=\mathcal{L}^{0}\left(X_{t}^{0}, \mathcal{F}_{t}\right)$ for random closed sets $\left(X_{t}^{0}\right)_{t=0, \ldots, T}$ such that $X_{t}^{0} \cap\left(-K_{t}\right)=\{0\}$ a.s. for all $t$. In the conical case, the latter condition is equivalent to $\operatorname{int}\left(X_{t}^{0}\right)^{*} \neq \varnothing$ for all 
$t$, and, under $\left(\mathrm{NA}^{\mathrm{s}}\right)$,

$$
\mathrm{A}_{t, T}=\sum_{s=t}^{T} \mathcal{L}^{0}\left(-X_{s}^{0}, \mathcal{F}_{s}\right), \quad 0 \leqslant t \leqslant T,
$$

where $X_{t}^{0}$ is a strictly proper random closed convex cone for all $t$.

Proof. Assume $\left(\mathrm{NA}^{\mathrm{s}}\right)$, so that Theorem 7.3 applies. Let $-g_{t} \in \mathcal{L}^{0}\left(X_{t}^{0} \cap\right.$ $\left.\left(-K_{t}\right), \mathcal{F}_{t}\right)$. Then $g_{t} \in K_{t}$ a.s., and there exist $k_{u} \in \mathcal{L}^{0}\left(K_{u}, \mathcal{F}_{t}\right), u=$ $t, \ldots, T$, and $\tilde{g}_{T} \in \mathcal{L}^{0}\left(K_{T}, \mathcal{F}_{T}\right)$, such that $-g_{t}-k_{t}-k_{t+1}-\cdots-k_{T}=\tilde{g}_{T}$. Since (SNA) holds, $g_{t}+k_{t}=0$, and $g_{t}=0$. The reverse implication is trivial. In the conical case, since $K_{t}+K_{t}=K_{t}$ for all $t \leqslant T$, (7.4) follows from the inclusions

$$
K_{t} \subset X_{t}^{0} \subset K_{t}+\cdots+K_{T}, \quad t \leqslant T .
$$

Note that $X_{T}^{0}=K_{T}$ is strictly proper by assumption. Since

$$
X_{t-1}^{0}=K_{t-1}+\mathbf{m}\left(X_{t}^{0} \mid \mathcal{F}_{t-1}\right) \subset K_{t-1}+X_{t}^{0},
$$

the induction argument yields that $X_{t}^{0} \subset K_{t}+\cdots+K_{T}$. Since (SNA) holds under $\left(\mathrm{NA}^{\mathrm{s}}\right), X_{t}^{0}$ is strictly proper for all $t$.

By [17, Lemma 5.1.2], $\left(\mathrm{NA}^{\mathrm{s}}\right)$ holds if and only if $\Xi_{t}^{0}$ is closed and $\Xi_{t}^{0}=\mathcal{L}^{0}\left(X_{t}^{0}, \mathcal{F}_{t}\right)$ with

$$
\operatorname{int} K_{t}^{*} \cap \operatorname{int}\left(X_{t}^{0}\right)^{*}=\operatorname{int} K_{t}^{*} \cap \operatorname{int} \mathbf{m}\left(X_{t+1}^{0} \mid \mathcal{F}_{t}\right)^{*} \neq \varnothing, \quad t \leqslant T .
$$

Finally, observe that

$$
\operatorname{int} K_{t}^{*} \cap \operatorname{int} \mathbf{m}\left(X_{t+1}^{0} \mid \mathcal{F}_{t}\right)^{*}=\operatorname{int} K_{t}^{*} \cap \mathbf{m}\left(X_{t+1}^{0} \mid \mathcal{F}_{t}\right)^{*}=\operatorname{int}\left(X_{t}^{0}\right)^{*} .
$$

Equation (7.4) means that, in the superhedging problem, we may replace solvency sets $K_{t}$ with $X_{t}^{0}$. The solvency sets $\left(X_{t}^{0}\right)_{t=0, \ldots, T}$ satisfies (NA2) condition by Lemma 7.2 , which is generally required to obtain a dual characterisation of the superhedging prices, see Condition $\mathbf{B}$ equivalent to (NA2) in [17, Sec. 3.6.3]. Therefore, $\left(\mathrm{NA}^{\mathrm{s}}\right)$ suffices for [17, Th. 3.6.3] to hold provided that we consider the consistent price systems associated to $\left(X_{t}^{0}\right)_{t=0, \ldots, T}$.

Now consider (NRA) and (NARA) condition for the chosen acceptance sets. Assume that the solvency sets are conical and satisfy $K_{t}^{*} \backslash\{0\} \subset \operatorname{int} \mathbb{R}_{+}^{d}$ which is the case if and only if $\mathbb{R}_{+}^{d} \backslash\{0\} \subset \operatorname{int} K_{t}^{\infty}$, see $\left[17\right.$, Section 3.2.1]. In that case, since $\hat{\Xi}_{t}^{0}=\operatorname{cl}_{0}\left(\hat{\Xi}_{t}^{0}\right)=\operatorname{cl}_{0}\left(\Xi_{t}^{0}\right)=\Xi_{t}^{0}$, (NRA) and (NARA) are equivalent by Proposition 6.5. Denote by $\mathrm{A}_{t, T}^{p}(\mathrm{Q})$ and $\overline{\mathrm{A}}_{t, T}^{p}(\mathrm{Q})$ for $p \in[1, \infty]$ the variants of $\mathrm{A}_{t, T}^{p}$ and $\overline{\mathrm{A}}_{t, T}^{p}$ when the reference probability measure is $\mathrm{Q}$. Let $q \geqslant 1$ denote the conjugate of $p \geqslant 1$, so that $p^{-1}+q^{-1}=1$.

Proposition 7.5. The following statements are equivalent. 
(i) $\overline{\mathrm{A}}_{t, T}^{p}(\mathrm{Q}) \cap \mathcal{L}^{0}\left(\mathbb{R}_{+}^{d}, \mathcal{F}_{t}\right)=\{0\}$, for all $t \leqslant T-1, p \in[1, \infty)$ and $\mathrm{Q} \sim \mathrm{P}$.

(ii) $\mathcal{M}_{t, T}^{q, w}(\mathrm{Q}) \neq \varnothing$ for every $t \leqslant T-1, \mathrm{Q} \sim \mathrm{P}$ and $p \in[1, \infty)$.

(iii) (NARA).

(iv) $\mathcal{M}_{t, T}^{\infty, w}(\mathrm{P}) \neq \varnothing$ for every $t \leqslant T-1$.

(v) $\mathcal{M}_{t, T}^{1, w}(\mathrm{P}) \neq \varnothing$ for every $t \leqslant T-1$.

Proof. By Theorem 6.10, (v) and (iii) are equivalent. We deduce the equivalence of (ii) and (iv) by following the proof of [17, Lemma 3.2.4], which makes it possible to construct a (weakly)-consistent price system (see Definition 6.9) from any consistent price system in $\mathcal{L}^{1}$. In particular, (v) implies (iv) and, clearly, (iv) implies (v). Then (iii) implies (ii), i.e. (NARA) holds for $Q$ in place of $P$. Using Theorem 6.6, we deduce that (i) holds. At last, (i) implies (NARA) by Theorem 6.6.

\section{Arbitrage With expeCtation as the Risk measure}

Assume that $p=1$ and

$$
\mathcal{C}_{t, s} \cap \mathcal{L}_{\mathcal{F}_{t}}^{1}\left(\mathbb{R}_{+}^{d}, \mathcal{F}_{s}\right)=\left\{\gamma \in \mathcal{L}_{\mathcal{F}_{t}}^{1}\left(\mathbb{R}^{d}, \mathcal{F}_{s}\right): \mathrm{E}^{g}\left(\gamma \mid \mathcal{F}_{t}\right) \geqslant 0\right\}
$$

In other words, the acceptable positions are those having non-negative generalised conditional expectation. The generalised conditional expectation is well defined for each $\xi \in \overline{\mathcal{L}}_{\mathcal{H}}^{p}\left(\mathbb{R}^{d}, \mathcal{F}\right)$ by letting $\mathrm{E}^{g}(\xi \mid \mathcal{H})=$ $\mathrm{E}^{g}\left(\xi^{\prime} \mid \mathcal{H}\right)+\mathrm{E}\left(\xi^{\prime \prime} \mid \mathcal{H}\right)$, where the second summand may be infinite. Then

$$
\mathrm{R}_{t, s}^{0}(X)=\left\{\mathrm{E}^{g}\left(\gamma \mid \mathcal{F}_{t}\right): \gamma \in \overline{\mathcal{L}}_{\mathcal{F}_{t}}^{1}\left(X, \mathcal{F}_{s}\right)\right\}
$$

and $\mathrm{R}_{t, s}(X)=\mathrm{E}^{g}\left(X \mid \mathcal{F}_{t}\right)$ is the generalised conditional expectation.

Lemma 3.14 yields that

$$
\begin{aligned}
\hat{\Xi}_{T-1}^{\xi} & =\mathcal{L}^{0}\left(K_{T-1}, \mathcal{F}_{T-1}\right)+\mathcal{L}^{0}\left(\mathrm{E}\left(\xi+K_{T} \mid \mathcal{F}_{T-1}\right), \mathcal{F}_{T-1}\right) \\
& =\mathcal{L}^{0}\left(K_{T-1}, \mathcal{F}_{T-1}\right)+\mathrm{E}\left(\xi \mid \mathcal{F}_{T-1}\right)+\mathcal{L}^{0}\left(\mathrm{E}\left(K_{T} \mid \mathcal{F}_{T-1}\right), \mathcal{F}_{T-1}\right) . \\
& =\mathcal{L}^{0}\left(X_{T-1}^{\xi}, \mathcal{F}_{T-1}\right),
\end{aligned}
$$

where

$$
X_{T-1}^{\xi}=K_{T-1}+\mathrm{E}\left(\xi \mid \mathcal{F}_{T-1}\right)+\mathrm{E}\left(K_{T} \mid \mathcal{F}_{T-1}\right)
$$

is $\mathcal{F}_{T-1}$-measurable by Lemma 2.8. Therefore,

$$
\begin{aligned}
\mathrm{R}_{T-2, T-1}\left(\hat{\Xi}_{T-1}^{\xi}\right) & =\mathrm{E}\left(X_{T-1}^{\xi} \mid \mathcal{F}_{T-2}\right) \\
& =\mathrm{E}\left(\xi \mid \mathcal{F}_{T-2}\right)+\mathrm{E}\left(K_{T-1}+\mathrm{E}\left(K_{t} \mid \mathcal{F}_{T-1}\right) \mid \mathcal{F}_{T-2}\right), \\
& =\mathrm{E}\left(\xi \mid \mathcal{F}_{T-2}\right)+\mathrm{E}\left(K_{T}+K_{T-1} \mid \mathcal{F}_{T-2}\right) .
\end{aligned}
$$


Since $\mathrm{R}_{T-2, T-1}^{0}\left(\Xi_{T-1}^{\xi}\right)$ is the set of $\mathrm{E}^{g}\left(k_{T-1}+\mathrm{E}^{g}\left(\xi+k_{T} \mid \mathcal{F}_{T-1}\right) \mid \mathcal{F}_{T-2}\right)$ for $k_{T-1} \in \mathcal{L}^{0}\left(K_{T-1}, \mathcal{F}_{T-1}\right)$ and $k_{T} \in \mathcal{L}_{\mathcal{F}_{T-1}}^{1}\left(K_{T}, \mathcal{F}_{T}\right)$, we deduce that $\mathrm{R}_{T-2, T-1}\left(\hat{\Xi}_{T-1}^{\xi}\right) \subset \mathrm{R}_{T-2, T-1}\left(\Xi_{T-1}^{\xi}\right)$. Moreover, it is trivial that

$$
\mathrm{R}_{T-2, T-1}^{0}\left(\Xi_{T-1}^{\xi}\right) \subset \mathrm{R}_{T-2, T-1}^{0}\left(\hat{\Xi}_{T-1}^{\xi}\right),
$$

so that $\mathrm{R}_{T-2, T-1}\left(\hat{\Xi}_{T-1}^{\xi}\right) \subset \mathrm{R}_{T-2, T-1}\left(\Xi_{T-1}^{\xi}\right)$. Therefore, $\mathrm{R}_{T-2, T-1}\left(\hat{\Xi}_{T-1}^{\xi}\right)=$ $\mathrm{R}_{T-2, T-1}\left(\Xi_{T-1}^{\xi}\right)$. Continuing recursively,

$$
\begin{aligned}
\hat{\Xi}_{T-2}^{\xi}= & \mathcal{L}^{0}\left(K_{T-2}, \mathcal{F}_{T-2}\right)+\mathcal{L}^{0}\left(\mathrm{E}\left(X_{T-1}^{\xi} \mid \mathcal{F}_{T-2}\right), \mathcal{F}_{T-2}\right) \\
= & \mathcal{L}^{0}\left(K_{T-2}, \mathcal{F}_{T-2}\right)+\mathrm{E}\left(\xi \mid \mathcal{F}_{T-2}\right) \\
& \quad+\mathcal{L}^{0}\left(\mathrm{E}\left(K_{T-1}+\mathrm{E}\left(K_{t} \mid \mathcal{F}_{T-1}\right) \mid \mathcal{F}_{T-2}\right), \mathcal{F}_{T-2}\right) \\
= & \mathcal{L}^{1}\left(X_{T-2}^{\xi}, \mathcal{F}_{T-2}\right),
\end{aligned}
$$

where

$$
X_{T-2}^{\xi}=K_{T-2}+\mathrm{E}\left(\xi \mid \mathcal{F}_{T-2}\right)+\mathrm{E}\left(K_{T}+K_{T-1} \mid \mathcal{F}_{T-2}\right),
$$

so that $\hat{\Xi}_{t}^{\xi}=\mathcal{L}^{0}\left(X_{t}^{\xi}, \mathcal{F}_{t}\right)$ with

$$
X_{t}^{\xi}=K_{t}+\mathrm{E}\left(\xi \mid \mathcal{F}_{t}\right)+\mathrm{E}\left(K_{T}+K_{T-1}+\cdots+K_{t+1} \mid \mathcal{F}_{t}\right) .
$$

Notice that $X_{t}^{\xi}=X_{t}^{0}+\mathrm{E}\left(\xi \mid \mathcal{F}_{t}\right)$, i.e. $X_{t}^{0}$ determines all superhedging prices. Furthermore,

$$
\mathrm{R}_{t, t+1}\left(\Xi_{t+1}^{0}\right)=\mathrm{E}^{g}\left(K_{t+1}+\cdots+K_{T} \mid \mathcal{F}_{t}\right),
$$

and

$$
X_{t}^{0}=K_{t}+\mathrm{E}^{g}\left(K_{t+1}+\cdots+K_{T} \mid \mathcal{F}_{t}\right) .
$$

Reformulating requirements from Definition 6.1, we arrive at the following result.

Proposition 8.1. For the risk arbitrage conditions formulated for the conditional expectation as the risk measure,

(i) If the solvency sets are strictly proper, (SNR) is equivalent to

$$
\mathrm{E}^{g}\left(K_{t+1}+\cdots+K_{T} \mid \mathcal{F}_{t}\right) \cap\left(-K_{t}\right)=\{0\}, \quad \text { a.s. }, t=0, \ldots, T-1 ;
$$

(ii) (NARA) is equivalent to

$$
\left(K_{t}+\mathrm{E}^{g}\left(K_{t+1}+\cdots+K_{T} \mid \mathcal{F}_{t}\right)\right) \cap \mathbb{R}_{-}^{d}=\{0\} \quad \text { a.s. }
$$

\section{Proposition 8.2.}

(i) (NRA) is equivalent to

$$
\begin{aligned}
\mathrm{A}_{t, T} \cap\left\{\xi \in \mathcal{L}_{\mathcal{F}_{t}}^{1}\left(\mathbb{R}^{d}, \mathcal{F}_{T}\right):\right. & \left.\mathrm{E}^{g}\left(\xi \mid \mathcal{F}_{t}\right) \in \mathbb{R}_{+}^{d} \text { a.s. }\right\} \\
& \subset\left\{\xi \in \mathcal{L}_{\mathcal{F}_{t}}^{1}\left(\mathbb{R}^{d}, \mathcal{F}_{T}\right): \mathrm{E}^{g}\left(\xi \mid \mathcal{F}_{t}\right)=0 \text { a.s. }\right\} .
\end{aligned}
$$


(ii) (NARA) is equivalent to

$$
\begin{aligned}
\overline{\mathrm{A}}_{t, T}^{p} \cap\left\{\xi \in \mathcal{L}^{p}\left(\mathbb{R}^{d}, \mathcal{F}_{T}\right)\right. & \left.: \mathrm{E}\left(\xi \mid \mathcal{F}_{t}\right) \in \mathbb{R}_{+}^{d} \text { a.s. }\right\} \\
& \subset\left\{\xi \in \mathcal{L}^{p}\left(\mathbb{R}^{d}, \mathcal{F}_{T}\right): \mathrm{E}\left(\xi \mid \mathcal{F}_{t}\right)=0 \text { a.s. }\right\}
\end{aligned}
$$

Proof. (i) Condition (8.3) trivially implies (NRA). Reciprocally, consider

$$
\xi \in \mathrm{A}_{t, T} \cap\left\{\xi \in \mathcal{L}_{\mathcal{F}_{t}}^{1}\left(\mathbb{R}^{d}, \mathcal{F}_{T}\right): \mathrm{E}^{g}\left(\xi \mid \mathcal{F}_{t}\right) \in \mathbb{R}_{+}^{d}\right\}
$$

Since

$$
\xi=\sum_{u=t+1}^{T}\left(\mathrm{E}^{g}\left(\xi \mid \mathcal{F}_{u}\right)-\mathrm{E}^{g}\left(\xi \mid \mathcal{F}_{u-1}\right)\right)+\mathrm{E}^{g}\left(\xi \mid \mathcal{F}_{t}\right),
$$

where $\mathrm{E}^{g}\left(\xi \mid \mathcal{F}_{u}\right)-\mathrm{E}^{g}\left(\xi \mid \mathcal{F}_{u-1}\right)$ is acceptable, we deduce that $\mathrm{E}^{g}\left(\xi \mid \mathcal{F}_{t}\right) \in$ $\mathrm{A}_{t, T} \cap \mathcal{L}^{0}\left(\mathbb{R}_{+}^{d}, \mathcal{F}_{t}\right)$. Thus, $\mathrm{E}^{g}\left(\xi \mid \mathcal{F}_{t}\right)=0$ a.s. under (NRA).

(ii) is shown similarly using Theorem 6.6.

Theorem 6.10 yields the following result.

Proposition 8.3. Suppose that the solvency sets $\left(K_{t}\right)_{t=0, \ldots, T}$ are cones. Let $q$ be the conjugate of $p$ that stems from the definition of the acceptance sets.

(1) (NARA) is equivalent to the fact that, for all $t$, there exists $Z \in \mathcal{M}_{t, T}^{q, w}(\mathrm{P})$, such that

$$
\mathrm{E}^{g}\left(\left\langle Z_{t+1}, \gamma_{t+1}\right\rangle \mid \mathcal{F}_{t}\right) \geqslant\left\langle Z_{t}, \mathrm{E}^{g}\left(\gamma_{t+1} \mid \mathcal{F}_{t}\right)\right\rangle \text { a.s. }
$$

for all $\gamma_{t+1} \in \mathcal{L}_{\mathcal{F}_{t}}^{p}\left(\mathbb{R}^{d}, \mathcal{F}_{t+1}\right)$.

(2) (SNR) is equivalent to the fact that, for all $t$, there exists $Z \in$ $\mathcal{M}_{t, T}^{q, w}(\mathrm{P})$ such that $(8.5)$ holds and $Z_{t} \in \mathcal{L}^{0}\left(\operatorname{int} K_{t}^{*}, \mathcal{F}_{t}\right)$.

\section{Application to the two-Dimensional MODEL}

Consider a financial market model composed of two assets. The first one has constant value 1 and the second one is a risky asset modelled by a bid-ask spread $Y_{t}=\left[S_{t}^{b}, S_{t}^{a}\right]$ such that $0<S_{t}^{b} \leqslant S_{t}^{a}$ a.s. for all $t \leqslant T$. This is Kabanov's model with the conical solvency set $K_{t}=C\left(Y_{t}\right)$, see Example 3.20.

Consider the acceptance sets from Section 7, so that the conditional core is the risk measure. Then $X_{T-1}^{0}$ is the sum of $K_{T-1}$ and $\mathbf{m}\left(X_{T}^{0}, \mathcal{F}_{T-1}\right)=C\left(\mathbf{M}\left(Y_{T} \mid \mathcal{F}_{T-1}\right)\right)$. Since $X_{T-1}^{0}$ is a random closed set, iterating this argument yields that $X_{t}^{0}=C\left(\tilde{Y}_{t}\right)$ for $t=0, \ldots, T$, where $\tilde{Y}_{T}=Y_{T}$ and

$$
\tilde{Y}_{t}=\mathbf{M}\left(\tilde{Y}_{t+1} \mid \mathcal{F}_{t}\right) \cap Y_{t}, \quad t=T-1, \ldots, 0 .
$$


Note that we do not make any no arbitrage assumption to obtain $X_{t}^{0}$. Observe that $\tilde{Y}_{t}=\left[\tilde{S}_{t}^{b}, \tilde{S}_{t}^{a}\right]$, where $\tilde{S}_{T}^{a}=S_{T}^{a}, \tilde{S}_{T}^{b}=S_{T}^{b}$, and

$$
\tilde{S}_{t}^{a}=S_{t}^{a} \wedge \operatorname{ess}_{\sup _{\mathcal{F}_{t}}} \tilde{S}_{t+1}^{a}, \quad \tilde{S}_{t}^{b}=S_{t}^{b} \vee \operatorname{ess} \inf _{\mathcal{F}_{t}} \tilde{S}_{t+1}^{b}
$$

for $t=T, \ldots, 1$. Since $0<\tilde{S}_{t}^{b} \leqslant \tilde{S}_{t}^{a}$ a.s. for all $t$, (NRA) always holds. By Definition 6.1 and Lemma 6.8, we easily deduce the following result.

\section{Theorem 9.1.}

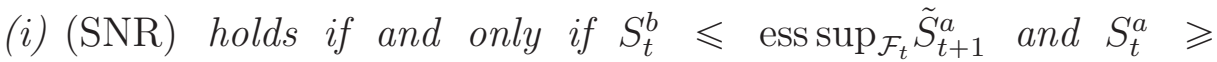
$\operatorname{ess}_{\inf } \tilde{\mathcal{F}}_{t} \tilde{S}_{t+1}^{b}$ a.s. with strict inequalities when $S_{t}^{b}<S_{t}^{a}$, for all $t \leqslant T-1$.

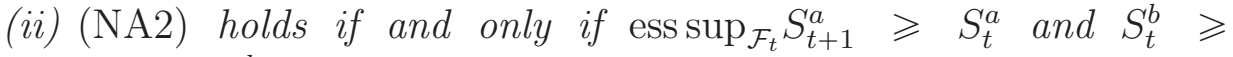
$\operatorname{essinf}_{\mathcal{F}_{t}} S_{t+1}^{b}$ a.s. for all $t \leqslant T-1$.

Remark 9.2. $\left(\mathrm{NA}^{\mathrm{s}}\right)$ is equivalent to (SNR) in the proper case but also to the existence of a strictly consistent price system, see [17, Th. 3.2.2]. In the two asset case, the Grigoriev theorem, see [17, Th. 3.2.15] and [11], asserts that $\left(\mathrm{NA}^{\mathrm{s}}\right)$ is equivalent to the existence of a (possibly nonstrict) consistent price system, i.e. the existence of a martingale $Z_{t}$ with respect to a probability measure $\mathrm{Q}$ equivalent to $\mathrm{P}$ such that $S_{t}^{b} \leqslant Z_{t} \leqslant$ $S_{t}^{a}$ for all $t$. By Theorem 9.1, the existence of a consistent price system, i.e. $\left(\mathrm{NA}^{\mathrm{s}}\right)$, implies $(\mathrm{SNR})$. Indeed, by Theorem A.2, $\operatorname{ess}_{\sup }{\tilde{S_{\mathcal{F}}}}_{t+1}^{a} \geqslant$ $\mathrm{E}_{\mathrm{Q}}\left(Z_{t+1} \mid \mathcal{F}_{t}\right) \geqslant S_{t}^{b}$ and similarly $\operatorname{ess} \inf _{\mathcal{F}_{t}} \tilde{S}_{t+1}^{b} \leqslant \mathrm{E}_{\mathrm{Q}}\left(Z_{t+1} \mid \mathcal{F}_{t}\right) \leqslant S_{t}^{a}$, the inequalities being strict when $S_{t}^{b}<S_{a}^{b}$.

Corollary 9.3. If there exist probability measures $\mathrm{Q}^{a}, \mathrm{Q}^{b}$ which are equivalent to $\mathrm{P}$, such that $S^{a}$ is a $\mathrm{Q}^{a}$-submartingale and $S^{b}$ is a $\mathrm{Q}^{b}$ supermartingale, then (NA2) holds.

The condition in the following corollary means that $\delta_{t}^{b}=S_{t}^{b} / S_{t-1}^{b}$ and $\delta_{t}^{a}=S_{t}^{a} / S_{t-1}^{a}$ admit conditional full supports on $\mathbb{R}_{+}$for all $t=1, \ldots, T$.

Corollary 9.4. If

$$
\mathrm{P}\left(\delta_{t}^{b} \leqslant c \mid \mathcal{F}_{t-1}\right) \mathrm{P}\left(\delta_{t}^{a} \geqslant c \mid \mathcal{F}_{t-1}\right)>0, \quad \text { a.s. }
$$

for all $t=1, \ldots, T$ and all $c>0$, then (NA2) holds.

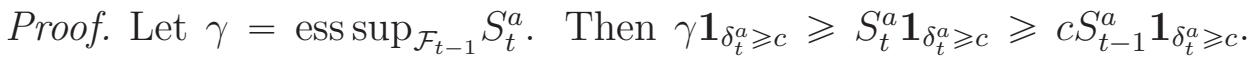
Taking the conditional expectation yields

$$
\gamma \mathrm{P}\left(\delta_{t}^{a} \geqslant c \mid \mathcal{F}_{t-1}\right) \geqslant c S_{t-1}^{a} \mathrm{P}\left(\delta_{t}^{a} \geqslant c \mid \mathcal{F}_{t-1}\right) .
$$

Then $\gamma \geqslant c S_{t-1}^{a}$, and letting $c \rightarrow \infty$ yields that $\gamma=+\infty$ a.s. Similarly, ess inf $_{\mathcal{F}_{t-1}} S_{t}^{b}=0$ a.s., and Theorem 9.1(ii) applies. 
Now switch to the setting of Section 8 with the acceptance sets given by (8.1). By Lemma 3.19,

$$
X_{T-1}^{0}=K_{T-1}+\mathrm{E}\left(K_{T} \mid \mathcal{F}_{T-1}\right)=C\left(Y_{T-1} \cap \mathbf{m}\left(Y_{T} \mid \mathcal{F}_{T-1}\right)\right) .
$$

Further, (8.2) yields that $X_{t}^{0}=C\left(\hat{Y}_{t}\right)$, where $\hat{Y}_{T}=Y_{T}$ and

$$
\hat{Y}_{t}=\mathbf{m}\left(\hat{Y}_{t+1} \mid \mathcal{F}_{t}\right) \cap Y_{t}, \quad t=T-1, \ldots, 0 .
$$

Then $\hat{Y}_{t}=\left[\hat{S}_{t}^{b}, \hat{S}_{t}^{a}\right]$, where $\hat{S}_{T}^{b}=S_{T}^{b}, \hat{S}_{T}^{a}=S_{T}^{a}$ and

$$
\hat{S}_{t}^{a}=\operatorname{essinf}_{\mathcal{F}_{t}}\left(\hat{S}_{t+1}^{a}\right) \wedge S_{t}^{a}, \quad \hat{S}_{t}^{b}=\operatorname{ess}_{\sup _{\mathcal{F}_{t}}}\left(\hat{S}_{t+1}^{b}\right) \vee S_{t}^{b},
$$

which can be compared to (9.1). Since $\hat{S}_{t}^{b}$ is not necessary smaller that $\hat{S}_{t}^{a}$, we use the convention that $C([b, a])=\mathbb{R}^{2}$ for $b>a$.

Theorem 9.5. With the acceptance sets given by (8.1),

(i) (SNR) is equivalent to the fact that $S_{t}^{b}<\operatorname{essinf}_{\mathcal{F}_{t}}\left(\hat{S}_{t+1}^{a}\right)$, and

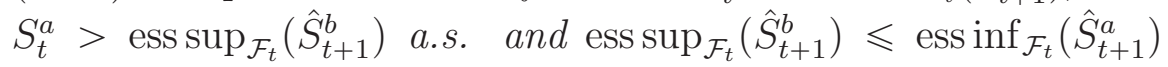
for all $t \leqslant T-1$;

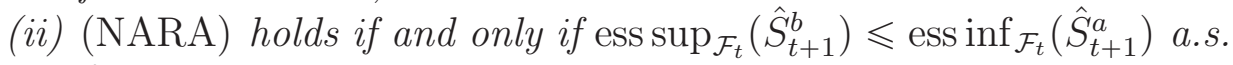
for all $t$

(iii) (NRA2) holds if and only if $\mathbf{m}\left(Y_{s} \mid \mathcal{F}_{t-1}\right) \supset \mathbf{m}\left(Y_{t} \mid \mathcal{F}_{t-1}\right)$ for all $s \geqslant t \geqslant 0$, and $K_{t}+\mathrm{E}\left(K_{t+1} \mid \mathcal{F}_{t}\right) \subset K_{t}+\mathcal{C}_{t-1, t}$ a.s., equivalently

$$
\begin{aligned}
\left\{\mathrm{E}\left(x_{t} \mid \mathcal{F}_{t-1}\right):\right. & \left.x_{t} \in \mathcal{L}^{1}\left(X_{t}^{0}, \mathcal{F}_{t}\right)\right\} \\
& \subset\left\{\mathrm{E}\left(k_{t} \mid \mathcal{F}_{t-1}\right): k_{t} \in \mathcal{L}^{1}\left(K_{t}, \mathcal{F}_{t}\right)\right\}, \quad t=0, \ldots, T .
\end{aligned}
$$

Proof. (i), (ii) It suffices to apply Proposition 8.1, recall that $S_{t}^{b} \leqslant S_{t}^{a}$.

(iii) Suppose that $(\mathrm{NRA2})$ holds. So, $\mathrm{E}\left(X_{t}^{0} \mid \mathcal{F}_{t-1}\right) \subset \mathrm{E}\left(K_{t} \mid \mathcal{F}_{t-1}\right)$, $t \geqslant 1$, by Lemma 6.8. Since $K_{t} \subset X_{t}^{0}, \mathrm{E}\left(X_{t}^{0} \mid \mathcal{F}_{t-1}\right)=\mathrm{E}\left(K_{t} \mid \mathcal{F}_{t-1}\right)$ for all $t \geqslant 1$. Since $\mathrm{E}\left(K_{u} \mid \mathcal{F}_{t-1}\right) \subset \mathrm{E}\left(X_{t}^{0} \mid \mathcal{F}_{t-1}\right)$ by (8.2) if $u \geqslant t$, we deduce that $\mathrm{E}\left(K_{u} \mid \mathcal{F}_{t-1}\right) \subset \mathrm{E}\left(K_{t} \mid \mathcal{F}_{t-1}\right)$ a.s., i.e. $\mathbf{m}\left(Y_{u} \mid \mathcal{F}_{t-1}\right) \supset \mathbf{m}\left(Y_{t} \mid \mathcal{F}_{t-1}\right)$, for all $u \geqslant t$. Therefore, $X_{t}^{0}=K_{t}+\mathrm{E}\left(X_{t+1}^{0} \mid \mathcal{F}_{t}\right)=K_{t}+\mathrm{E}\left(K_{t+1} \mid \mathcal{F}_{t}\right)$, a.s. Reciprocally, we may show by induction that $X_{t}^{0}=K_{t}+\mathrm{E}\left(K_{t+1} \mid \mathcal{F}_{t}\right)$ a.s. for all $t \leqslant T-1$ with (8.2) since this holds for $t=T-1$. Therefore (NRA2) holds.

(NRA2) immediately implies (9.3). Reciprocally, assume (9.3) and consider $x_{t} \in \mathcal{L}^{1}\left(X_{t}^{0}, \mathcal{F}_{t}\right)$. Then $x_{t}=\eta_{t}+\mathrm{E}\left(x_{t} \mid \mathcal{F}_{t-1}\right)$, where $\eta_{t}=$ $x_{t}-\mathrm{E}\left(x_{t} \mid \mathcal{F}_{t-1}\right) \in \mathcal{C}_{t-1, t}$ and, by assumption, $\mathrm{E}\left(x_{t} \mid \mathcal{F}_{t-1}\right)=\mathrm{E}\left(k_{t} \mid \mathcal{F}_{t-1}\right)$ for some $k_{t} \in \mathcal{L}^{1}\left(K_{t}, \mathcal{F}_{t}\right)$. Then $\mathrm{E}\left(k_{t} \mid \mathcal{F}_{t-1}\right)=\tilde{\eta}_{t}+k_{t}$, where $\tilde{\eta}_{t}=$ $\mathrm{E}\left(k_{t} \mid \mathcal{F}_{t-1}\right)-k_{t} \in \mathcal{C}_{t-1, t}$. Therefore, $x_{t}=\eta_{t}+\tilde{\eta}_{t}+k_{t} \in K_{t}+\mathcal{C}_{t-1, t}$, i.e. (NRA2) holds by Lemma 6.8 .

Corollary 9.6. If (NRA2) holds, then $X_{t}^{0}=K_{t}+\mathrm{E}\left(K_{t+1} \mid \mathcal{F}_{t}\right)$ for all $t \leqslant T-1$. 


\section{Appendix A. Conditional essential supremum}

Let $\Xi \subset \mathcal{L}^{0}(\mathbb{R}, \mathcal{F})$ be a (possibly uncountable) family of real-valued $\mathcal{F}$-measurable random variables and let $\mathcal{H}$ be a sub- $\sigma$-algebra of $\mathcal{F}$. The following result is well known, see e.g. [10, Appendix A.5] and a similar result holds for the conditional essential infimum.

Theorem A.1. For any family $\Xi$ of random variables, there exits a

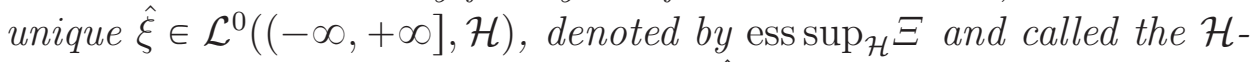
conditional supremum of $\Xi$, such that $\hat{\xi} \geqslant \xi$ a.s. for all $\xi \in \Xi$ and $\eta \geqslant \xi$ a.s. for $\eta \in \mathcal{L}^{0}((-\infty,+\infty], \mathcal{H})$ and all $\xi \in \Xi$ implies $\eta \geqslant \hat{\xi}$ a.s.

If $\mathcal{H}$ is trivial, we recover the deterministic essential supremum and infimum of random variables. Let $\mathcal{Q}$ be the set of all absolutely continuous probability measures $Q$ with respect to $P$ and let $E_{Q}$ designate the expectation under $\mathrm{Q}$.

Theorem A.2. If $\Xi \subset \mathcal{L}^{0}\left(\mathbb{R}_{+}, \mathcal{F}\right)$ or $\Xi \subset \mathcal{L}^{\infty}(\mathbb{R}, \mathcal{F})$, then

$$
\operatorname{ess}_{\sup } \Xi=\operatorname{ess}_{\mathcal{H}} \sup _{\mathcal{F}}\left\{\mathrm{E}_{\mathrm{Q}}^{g}(\xi \mid \mathcal{H}), \xi \in \Xi, \mathrm{Q} \in \mathcal{Q}\right\} \text {. }
$$

Moreover, if $\Xi=\{\xi\}$ is a singleton, the family $\left\{\mathrm{E}_{\mathrm{Q}}^{g}(\xi \mid \mathcal{H}), \mathrm{Q} \in \mathcal{Q}\right\}$ is directed upwards, hence there exists a sequence $\mathrm{Q}^{n} \in \mathcal{Q}, n \geqslant 1$, such that $\mathrm{E}_{\mathrm{Q}^{n}}(\xi \mid \mathcal{H}) \uparrow \operatorname{ess} \sup _{\mathcal{H}} \Xi$ everywhere on $\Omega$.

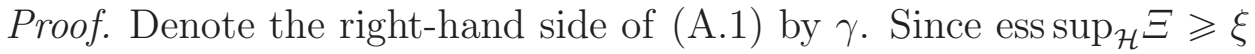

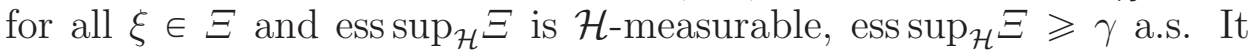
remains to show that $\gamma \geqslant \xi$ a.s. for all $\xi \in \Xi$. Assume without loss of generality that $\gamma<\infty$ a.s. and there exists $\xi \in \Xi$ such that $\gamma<\xi$ on a non null set $F$. Then $\mathrm{E}_{\mathrm{Q}}^{g}(\xi \mid \mathcal{H}) \mathbf{1}_{F}+\xi \mathbf{1}_{F^{c}} \leqslant \xi$, for measure $\mathrm{Q}$ with density $\mathbf{1}_{F}$, and the inequality is strict on $F$. By taking the conditional expectation and noticing that $\gamma<\infty$ a.s., $\mathrm{E}_{\mathbf{Q}}\left(\xi \mathbf{1}_{F^{c}} \mid \mathcal{H}\right) \leqslant \mathrm{E}_{\mathbf{Q}}(\xi \mid \mathcal{H}) \mathrm{E}_{\mathbf{Q}}\left(\mathbf{1}_{F^{c}} \mid \mathcal{H}\right)$, and the inequality is strict on $F$, contrary to $\mathrm{Q}\left(F^{c}\right)=0$.

Suppose that $\Xi=\{\xi\}$ is a singleton. Consider two mesures $\mathrm{Q}^{1}, \mathrm{Q}^{2} \in$ $\mathcal{Q}$ with densities $\alpha_{1}, \alpha_{2}$. Let $\mathrm{Q} \in \mathcal{Q}$ have density

$$
\alpha=c \alpha_{1} \mathbf{1}_{\mathrm{E}_{\mathrm{Q}^{1}}(\xi \mid \mathcal{H}) \geqslant \mathrm{E}_{\mathrm{Q}^{2}}(\xi \mid \mathcal{H})}+c \alpha_{2} \mathbf{1}_{\mathrm{E}_{\mathrm{Q}^{1}}(\xi \mid \mathcal{H})<\mathrm{E}_{\mathrm{Q}^{2}}(\xi \mid \mathcal{H})},
$$

where $c>0$ is the normalising constant and $\mathrm{E}_{\mathrm{P}}\left(c^{-1} \alpha\right)>0$. Since

$$
\mathrm{E}_{\mathrm{Q}}\left(\xi \mathbf{1}_{A}\right)=\mathrm{E}_{\mathrm{P}}\left(\alpha \xi \mathbf{1}_{A}\right) \geqslant \mathrm{E}_{\mathrm{Q}}\left(\mathrm{E}_{\mathrm{Q}^{i}}(\xi \mid \mathcal{H}) \mathbf{1}_{A}\right) \quad \text { a.s. }
$$

for every $A \in \mathcal{H}$, we have $\mathrm{E}_{\mathrm{Q}}(\xi \mid \mathcal{H}) \geqslant \mathrm{E}_{\mathrm{Q}^{i}}(\xi \mid \mathcal{H})$ a.s. for $i=1,2$.

\section{Appendix B. Generalised conditional expectation}

Definition B.1. Let $\mathcal{H}$ be a sub- $\sigma$-algebra of $\mathcal{F}$. The generalised conditional expectation $\mathrm{E}^{g}(\xi \mid \mathcal{H})$ of $\xi \in \mathcal{L}^{0}\left(\mathbb{R}^{d}, \mathcal{F}\right)$ is said to exist and 
$\xi$ belongs to the family $\xi \in \mathcal{L}_{\mathcal{H}}^{1}\left(\mathbb{R}^{d}, \mathcal{F}\right)$ if there exists an $\mathcal{H}$-measurable partition $\left(B_{i}\right)_{i \geqslant 1}$ such that $\xi \mathbf{1}_{B_{i}}$ is integrable for all $i \geqslant 1$. Then

$$
\mathrm{E}^{g}(\xi \mid \mathcal{H})=\sum_{i=1}^{\infty} \mathrm{E}\left(\xi \mathbf{1}_{B_{i}} \mid \mathcal{H}\right) \mathbf{1}_{B_{i}}
$$

It is easy to see that the generalised conditional expectation does not depend on the chosen partition.

Theorem B.2. We have $\xi \in \mathcal{L}_{\mathcal{H}}^{1}\left(\mathbb{R}^{d}, \mathcal{F}\right)$ if and only if $\mathrm{E}(\|\xi\| \mid \mathcal{H})<\infty$ a.s. Moreover, $\mathrm{E}^{g}(\xi \mid \mathcal{H})=\mathrm{E}\left(\xi^{+} \mid \mathcal{H}\right)-\mathrm{E}\left(\xi^{-} \mid \mathcal{H}\right)$ for all $\xi \in \mathcal{L}_{\mathcal{H}}^{1}\left(\mathbb{R}^{d}, \mathcal{F}\right)$, where $\xi^{+}=\xi \vee 0$ and $\xi^{-}=-(\xi \wedge 0)$ with maximum and minimum applied coordinatewisely.

Proof. Without loss of generality, assume that $d=1$. Suppose that $\mathrm{E}(|\xi| \mid \mathcal{H})<\infty$ a.s., in particular, $\mathrm{E}\left(\xi^{+} \mid \mathcal{H}\right)<\infty$ and $\mathrm{E}\left(\xi^{-} \mid \mathcal{H}\right)<\infty$ a.s. Then $B_{n}=\{\mathrm{E}(|\xi| \mid \mathcal{H}) \in[n, n+1)\}, n \geqslant 0$, build a partition of $\Omega$. The reverse implication is trivial.

Define

$$
\|\xi\|_{p, \mathcal{H}}= \begin{cases}\mathrm{E}\left(\|\xi\|^{p} \mid \mathcal{H}\right)^{1 / p}, & p \in[1, \infty), \\ {\operatorname{ess} \sup _{\mathcal{H}}\|\xi\|,} \|=\infty .\end{cases}
$$

The family $\mathcal{L}_{\mathcal{H}}^{p}\left(\mathbb{R}^{d}, \mathcal{F}\right)$ for $p \in[1, \infty]$ consists of random vectors from $\mathcal{L}^{0}\left(\mathbb{R}^{d}, \mathcal{F}\right)$ such that $\|\xi\|_{p, \mathcal{H}}$ is a.s. finite. Observe that $\mathcal{L}^{0}\left(\mathbb{R}^{d}, \mathcal{H}\right)=$ $\mathcal{L}_{\mathcal{H}}^{p}\left(\mathbb{R}^{d}, \mathcal{H}\right)$ for all $p \in[1, \infty]$. The family $\mathcal{L}_{\mathcal{H}}^{p}\left(\mathbb{R}^{d}, \mathcal{F}\right)$ is an $\mathcal{L}^{0}(\mathbb{R}, \mathcal{H})$ module, see [8]. We endow the space $\mathcal{L}_{\mathcal{H}}^{p}\left(\mathbb{R}^{d}, \mathcal{F}\right)$ with the topology by assuming that $\xi_{n}$ converges to $\xi$ if $\left\|\xi_{n}-\xi\right\|_{p, \mathcal{H}} \rightarrow 0$ in probability if $p \in[1, \infty)$. If $p=\infty$, we use the bounded convergence in probability, meaning that ess $\sup _{\mathcal{H}}\left\|\xi_{n}\right\|$ is bounded and $\left\|\left(\xi_{n}-\xi\right) \wedge 1\right\|_{1, \mathcal{H}} \rightarrow 0$ in probability as $n \rightarrow \infty$. By conditional normalisation, we may easily show the following result.

Lemma B.3. Random vector $\xi \in \mathcal{L}^{0}\left(\mathbb{R}^{d}, \mathcal{F}\right)$ belongs to $\mathcal{L}_{\mathcal{H}}^{p}\left(\mathbb{R}^{d}, \mathcal{F}\right)$ if and only if $\xi=\gamma \tilde{\xi}$, where $\gamma \in \mathcal{L}^{0}([1, \infty), \mathcal{H})$ and $\tilde{\xi} \in \mathcal{L}^{p}\left(\mathbb{R}^{d}, \mathcal{F}\right)$.

The next result follows from the conditional dominated convergence theorem.

Lemma B.4 (Generalised conditional dominated convergence theorem). Let $\left(\xi^{n}\right)_{n \geqslant 1}$ be a sequence from $\mathcal{L}_{\mathcal{H}}^{p}\left(\mathbb{R}^{d}, \mathcal{F}\right)$ with $p \in[1, \infty)$ which converges a.s. to $\xi \in \mathcal{L}^{0}\left(\mathbb{R}^{d}, \mathcal{F}\right)$. If $\left\|\xi^{n}\right\| \leqslant \gamma$ a.s. for some $\gamma \in \mathcal{L}_{\mathcal{H}}^{p}\left(\mathbb{R}_{+}, \mathcal{F}\right)$ and all $n$, then $\xi^{n} \rightarrow \xi$ in $\mathcal{L}_{\mathcal{H}}^{p}\left(\mathbb{R}^{d}, \mathcal{F}\right)$.

Corollary B.5. The set $\mathcal{L}^{p}\left(\mathbb{R}^{d}, \mathcal{F}\right)$ is dense in $\mathcal{L}_{\mathcal{H}}^{p}\left(\mathbb{R}^{d}, \mathcal{F}\right)$ for all $p \in$ $[1, \infty]$. 
Lemma B.6. Let $\left(\xi^{n}\right)_{n \geqslant 1}$ be a sequence from $\mathcal{L}_{\mathcal{H}}^{p}\left(\mathbb{R}^{d}, \mathcal{F}\right)$ which converges to $\xi$ in $\mathcal{L}_{\mathcal{H}}^{p}\left(\mathbb{R}^{d}, \mathcal{F}\right)$. Then there exists a random $\mathcal{H}$-measurable sequence $\left(n_{k}\right)_{k \geqslant 1}$ of $\mathcal{H}$-measurable natural numbers, such that $\xi^{n_{k}} \in$ $\mathcal{L}_{\mathcal{H}}^{p}\left(\mathbb{R}^{d}, \mathcal{F}\right)$ and $\xi^{n_{k}} \rightarrow \xi$ a.s.

Proof. Since $\xi^{n} \rightarrow \xi$ in $\mathcal{L}_{\mathcal{H}}^{p}\left(\mathbb{R}^{d}, \mathcal{F}\right)$, we deduce that $\mathrm{E}\left(\left\|\xi^{m}-\xi\right\|^{p} \mid \mathcal{H}\right) \rightarrow 0$ a.s. as $m \rightarrow \infty$. Define $n_{0}=0$ and

$$
n_{k}=\inf \left\{n>n_{k-1}: \mathrm{E}\left(\left\|\xi^{i}-\xi\right\|^{p} \mid \mathcal{H}\right) \leqslant 2^{-p k} \text { for all } i \geqslant n\right\}, \quad k \geqslant 1 .
$$

Then $\mathrm{E}\left(\left\|\xi^{n_{k}}-\xi\right\|^{p} \mid \mathcal{H}\right) \leqslant 2^{-p k}$, so that $\mathrm{E}\left(\left\|\xi^{n_{k}}-\xi\right\|^{p}\right) \leqslant 2^{-p k}$. Therefore, $\left(\xi^{n_{k}}-\xi\right) \rightarrow 0$ in $\mathcal{L}^{p}\left(\mathbb{R}^{d}, \mathcal{F}\right)$ and almost surely for a subsequence. Observe that

$$
\mathrm{E}\left(\left\|\xi^{n_{k}}\right\| \mid \mathcal{H}\right)=\sum_{j \geqslant k} \mathrm{E}\left(\left\|\xi^{j}\right\| \mid \mathcal{H}\right) \mathbf{1}_{n_{k}=j}<\infty,
$$

so that $\xi^{n_{k}} \in \mathcal{L}_{\mathcal{H}}^{p}\left(\mathbb{R}^{d}, \mathcal{F}\right)$. The conclusion follows.

\section{REFERENCES}

[1] B. Acciaio and I. Penner. Dynamic risk measures. In Advanced mathematical methods for finance, pages 1-34. Springer, Heidelberg, 2011.

[2] I. Cascos and I. Molchanov. Multivariate risks and depth-trimmed regions. Finan. Stoch., 11:373-397, 2007.

[3] P. Cheridito, M. Kupper, and N. Vogelpoth. Conditional analysis on $\mathbb{R}^{d}$. In A. Hamel, F. Heyde, A. Löhne, B. Rudloff, and C. Schrage, editors, Set Optimization and Applications in Finance - The State of the Art. Springer, Berlin, 2015.

[4] A. S. Cherny and P. G. Grigoriev. Dilatation monotone risk measures are law invariant. Finance Stoch., 11:291-298, 2007.

[5] F. Delbaen. Monetary Utility Functions. Osaka University Press, Osaka, 2012.

[6] Z. Feinstein and B. Rudloff. A comparison of techniques for dymanic multivariate risk measures. In A. Hamel, F. Heyde, A. Löhne, B. Rudloff, and C. Schrage, editors, Set Optimization and Applications in Finance - The State of the Art. Springer, Berlin, 2015.

[7] Z. Feinstein and B. Rudloff. Multi-portfolio time consistency for set-valued convex and coherent risk measures. Finan. Stoch., 19:67-107, 2015.

[8] D. Filipović, M. Kupper, and N. Vogelpoth. Separation and duality in locally $L^{0}$-convex modules. J. Funct. Anal., 256:3996-4029, 2009.

[9] D. Filipović, M. Kupper, and N. Vogelpoth. Approaches to conditional risk. SIAM J. Financial Math., 3:402-432, 2012.

[10] H. Föllmer and A. Schied. Stochastic Finance. An Introduction in Discrete Time. De Gruyter, Berlin, 2 edition, 2004.

[11] P. G. Grigoriev. On low dimensional case in the fundamental asset pricing theorem with transaction costs. Statist. Decisions, 23:33-48, 2005.

[12] A. H. Hamel and F. Heyde. Duality for set-valued measures of risk. SIAM J. Finan. Engineering, 1:66-95, 2010.

[13] A. H. Hamel, B. Rudloff, and M. Yankova. Set-valued average value at risk and its computation. Math. Finan. Economics, 7:229-246, 2013. 
[14] C. Hess. Set-valued integration and set-valued probability theory: An overview. In E. Pap, editor, Handbook of Measure Theory, chapter 14, pages 617-673. Elsevier, 2002.

[15] C. Hess, R. Seri, and C. Choirat. Essential intersection and approximation results for robust optimization. J. Nonlinear Convex Anal., 15:979-1002, 2014.

[16] F. Hiai and H. Umegaki. Integrals, conditional expectations, and martingales of multivalued functions. J. Multiv. Anal., 7:149-182, 1977.

[17] Y. M. Kabanov and M. Safarian. Markets with Transaction Costs. Mathematical Theory. Springer, Berlin, 2009.

[18] M. Kaina and L. Rüschendorf. On convex risk measures on $L^{p}$-spaces. Math. Meth. Oper. Res., 69:475-495, 2009.

[19] E. Lépinette. Robust no arbitrage condition for continuous-time models with transaction costs. In M. Kijima, C. Hara, Y. Muromachi, H. Nakaoka, and K. Nishide, editors, Recent Advances in Financial Engineering, Proceedings of the Kier-Tmu international workshop on financial engineering, pages 69-82, Singapore, 2010. World Scientific.

[20] A. Löhne and B. Rudloff. An algorithm for calculating the set of superhedging portfolios and strategies in markets with transaction costs. Int. J. Theor. Appl. Finance, 17:1450012 (33 pages), 2014.

[21] I. Molchanov. Theory of Random Sets. Springer, London, 2005.

[22] I. Molchanov and I. Cascos. Multivariate risk measures: a constructive approach based on selections. Math. Finance, 2016. To appear.

[23] T. Pennanen and I. Penner. Hedging of claims with physical delivery under convex transaction costs. SIAM J. Finan. Engineering, 1:158-178, 2010.

[24] M. Rásonyi. New methods in the arbitrage theory in financial markets with transaction costs. In C. Donati-Martin, M. Émery, A. Rouault, and C. Stricker, editors, Séminaire de Probabilités XLI, volume 1934 of Lect. Notes Math., pages 455-462. Springer, Berlin, 2008.

[25] W. Schachermayer. The fundamental theorem of asset pricing under proportional transaction costs in finite discrete time. Math. Finance, 14:19-48, 2004.

[26] N. Vogelpoth. $L^{0}$-convex Analysis and Conditional Risk Measures. $\mathrm{PhD}$ thesis, Universität Wien, 2009.

Ceremade, Paris-Dauphine University, Place du Maréchal De Lattre De Tassigny, 75775 Paris cedex 16, France

E-mail address: emmanuel.lepinette@ceremade.dauphine.fr

University of Bern, Institute of Mathematical Statistics and ACtuarial Science, Sidlerstrasse 5, CH-3012 Bern, Switzerland

E-mail address: ilya.molchanov@stat.unibe.ch 